ARTICLE

https://doi.org/10.1038/s41467-019-10066-7

\title{
Inactivation of nuclear histone deacetylases by EP300 disrupts the MiCEE complex in idiopathic pulmonary fibrosis
}

\author{
Karla Rubio (1) et al."
}

Idiopathic pulmonary fibrosis (IPF) is a chronic, progressive, and highly lethal lung disease with unknown etiology and poor prognosis. IPF patients die within 2 years after diagnosis mostly due to respiratory failure. Current treatments against IPF aim to ameliorate patient symptoms and to delay disease progression. Unfortunately, therapies targeting the causes of or reverting IPF have not yet been developed. Here we show that reduced levels of miRNA lethal 7d (MIRLET7D) in IPF compromise epigenetic gene silencing mediated by the ribonucleoprotein complex MiCEE. In addition, we find that hyperactive EP300 reduces nuclear HDAC activity and interferes with MiCEE function in IPF. Remarkably, EP300 inhibition reduces fibrotic hallmarks of in vitro (patient-derived primary fibroblast), in vivo (bleomycin mouse model), and ex vivo (precision-cut lung slices, PCLS) IPF models. Our work provides the molecular basis for therapies against IPF using EP300 inhibition. 
diopathic pulmonary fibrosis (IPF) is the most common interstitial pulmonary disease showing a prevalence of 20 new cases per 100,000 persons per year ${ }^{1,2}$. Unfortunately, these numbers are rising due to increased lung injuries after exposure to air pollution, a consequence of industrialization ${ }^{3-5}$. A central event in IPF is the abnormal proliferation and migration of fibroblasts into the alveolar space after lung injury. Although under normal circumstances fibroblasts are important for wound healing and connective tissue production, their function in the fibrotic lung is out of control. It comes to formation of fibroblastic foci, which consist of highly proliferative fibroblasts, certain immune cells, and excessive extracellular matrix (ECM) protein deposition, such as fibronectin (FN1) and collagen (COL1A1). The consequences of these processes are disproportionate levels of scar tissue, alterations of the alveolar framework, changes in the lung epithelium structure, stiffening of the functional lung tissue, loss of the gas exchange function of the lung, and dramatically decreased oxygen saturation of the blood 2,6 .

Myofibroblasts are the key effector cell type that promotes remodeling of connective tissue after injury. Myofibroblasts are contractile-activated fibroblasts that express $\alpha$-smooth muscle actin (ACTA2) to facilitate wound closure ${ }^{7-10}$. After wound healing, myofibroblasts can dedifferentiate into resting cells such as lipofibroblasts ${ }^{9,11}$. However, persistent activation of myofibroblasts causes excessive deposition of ECM proteins in the interstitial space leading to fibroblastic foci ${ }^{5,12}$. The precise control of gene expression is essential for both expansions of activated fibroblasts after injury and dedifferentiation into resting fibroblasts after wound healing. During dedifferentiation of activated fibroblast into resting fibroblast, the balance between transcription of lineage-specific genes and repression of profibrotic genes allows resolution of fibroblastic foci. Transcriptional regulation of gene expression is closely linked to chromatin structure, which constitute the physiological template for all DNA-dependent biological processes in the cell nucleus. Chromatin structure is modulated by different processes including covalent modification of nucleotides on the DNA ${ }^{13}$, posttranslational histone modifications ${ }^{14,15}$, changes of nucleosome density by nucleosome remodeling or deposition/eviction ${ }^{16}$, changes in histone composition of nucleosomes ${ }^{17}$, activity of non-histone chromatin-associated proteins ${ }^{18,19}$, and gene regulation by noncoding RNAs (ncRNAs) including microRNAs (miRNAs, 21-25 nucleotides long) and long ncRNAs (>200 nucleotides long $)^{20}$. Beyond its association to several human diseases, miRNAs are considered key diagnostic markers as well as dynamic regulators of phase separation as the organizing principle for tridimensional nuclear architecture ${ }^{21}$. Increasing evidence of mature miRNAs in the nucleus suggests a novel function in sub-nuclear compartments of mammalian cells ${ }^{22-24}$. In our previous work ${ }^{25}$ we characterize a multicomponent RNA-protein complex containing miRNA-ncRNA duplexes, the exosome-associated protein C1D, the nuclear-specific exosome subunit EXOSC10 (also known as RRP6), and the histone methyl transferase EZH2 (enhancer of zeste homolog 2). We demonstrate that this ribonucleoprotein complex, which we called MiCEE (Mirlet7d-C1D-EXOSC10$\mathrm{EZH} 2$ ), mediates global genome organization and epigenetic silencing of bi-directionally expressed genes. In the present work we elucidate the clinical relevance of MiCEE using human lung tissue and primary fibroblasts, both isolated from control donors and IPF patients. The nuclear miRNA lethal 7d (MIRLET7D, also known as let-7d), is a functional component of $\mathrm{MiCEE}^{25}$. We demonstrate that reduced MIRLET7D levels in IPF compromised epigenetic silencing mediated by the MiCEE complex. In addition, we find that in control donors, deacetylation of histone 3 at lysine 27 (H3K27) mediated by histone deacetylase 1 and 2 $\left(\mathrm{HDAC1}\right.$ and HDAC2) ${ }^{26}$ anticipates methylation of the same residue (H3K27me3) during MiCEE-mediated heterochromatin formation. However, in IPF we detect hyperactive EP300 (E1A-binding protein p300, also known as P300) ${ }^{27}$, which inhibits nuclear HDAC1 and interferes with MiCEE function. Interestingly, we find reduced HDAC activity in the nucleus of IPF fibroblasts, which apparently is in contrast to previous reports $^{28-30}$ that propose the use of HDAC inhibitors as potential treatment against pulmonary fibrosis. Remarkably, results after EP300 inhibition support our model and demonstrate reduced fibrotic hallmarks of in vitro (patient-derived primary fibroblast), in vivo (bleomycin mouse model), and ex vivo (precision-cut lung slices, PCLS) IPF models. Our study provides the molecular basis toward more efficient therapies against IPF using EP300 inhibition.

\section{Results}

Reduced MIRLET7D in IPF compromises MiCEE complex function. Analysis of publically available RNA-sequencing (RNA-seq) data of lung tissue samples from IPF patients ${ }^{31}$ showed increased levels of fibrosis markers (Fig. 1a), including FN1, COL1A1, ACTA2, and snail family transcription repressors 1 (SNAI1, also known as Snail) and 2 (SNAI2, also known as Slug) ${ }^{17}$. KEGG (Kyoto Encyclopedia of Genes and Genomes) pathway analysis of the transcripts with increased expression in IPF patients (Fig. 1b, top) revealed statistically significant enrichment of pathways related to a fibrotic phenotype. Interestingly, we found a high positive correlation (Pearson's $R^{2}=0.9 ; P=0.0002$; Fig. 1b, bottom) between the sample-to-sample expression (measured as a $\log 2$ FC value in the RNA-seq data) of fibrosis markers and genes that we recently identified as targets of MIRLET7D in the cell nucleus (CHMP2B, MAFG, NOLC1, and PSMD3; further referred as MIRLET7D targets) ${ }^{25}$. To confirm these results, we analyzed the expression of mature MIRLET7D and its targets by TaqMan assay and quantitative reverse transcriptase PCR (qRT-PCR) in lung tissue samples from control (Ctrl; $n=5)$ and IPF ( $n=10)$ patients, respectively (Fig. 1c). We observed reduced MIRLET7D levels in IPF when compared with Ctrl human lung tissue, as previously reported ${ }^{32}$. Correlating with reduced MIRLET7D levels, we detected increased expression of MIRLET7D targets concomitant with high transcript levels of fibrosis markers. Our results confirmed that the recently identified MIRLET7D targets ${ }^{25}$ could be used as novel IPF markers.

In silico analysis of human MIRLET7D target loci (Supplementary Fig. 1a) revealed similar gene structures as in the mouse orthologs, which suggested transcriptional activity leading to the expression of ncRNA and corresponding mRNA from each locus $^{33,34}$. To determine whether the ribonucleoprotein complex $\mathrm{MiCEE}^{25}$, in which Mirlet7d is functionally relevant, mediates epigenetic silencing in humans as it does in mice, we performed various experiments using primary fibroblasts isolated from lung tissue from Ctrl $(n=3)$ and IPF $(n=3)$ patients (Supplementary Fig. 1b). Combined MIRLET7D-specific fluorescence in situ hybridization (FISH) and EXOSC10-specific immunostaining (Fig. 2a) revealed co-localization of MIRLET7D and EXOSC10 in specific regions of the nucleus of human primary Ctrl fibroblasts. In addition, we detected reduced MIRLET7D levels in the nucleus and cytosol of IPF fibroblasts, which were further confirmed by TaqMan assay-based expression analysis after cellular fractionation (Supplementary Fig. 1c). RNA-seq in primary fibroblasts (Supplementary Fig. 2a-c) confirmed the RNA-seq results from human lung tissue (Fig. 1a), i.e., increased levels of MIRLET7D targets in IPF fibroblasts concomitant with fibrosis markers (Supplementary Fig. 2c, left). In addition, alternative mapping of our RNA-seq data to NONCODE database (Supplementary Fig. 2c, right) revealed increased 

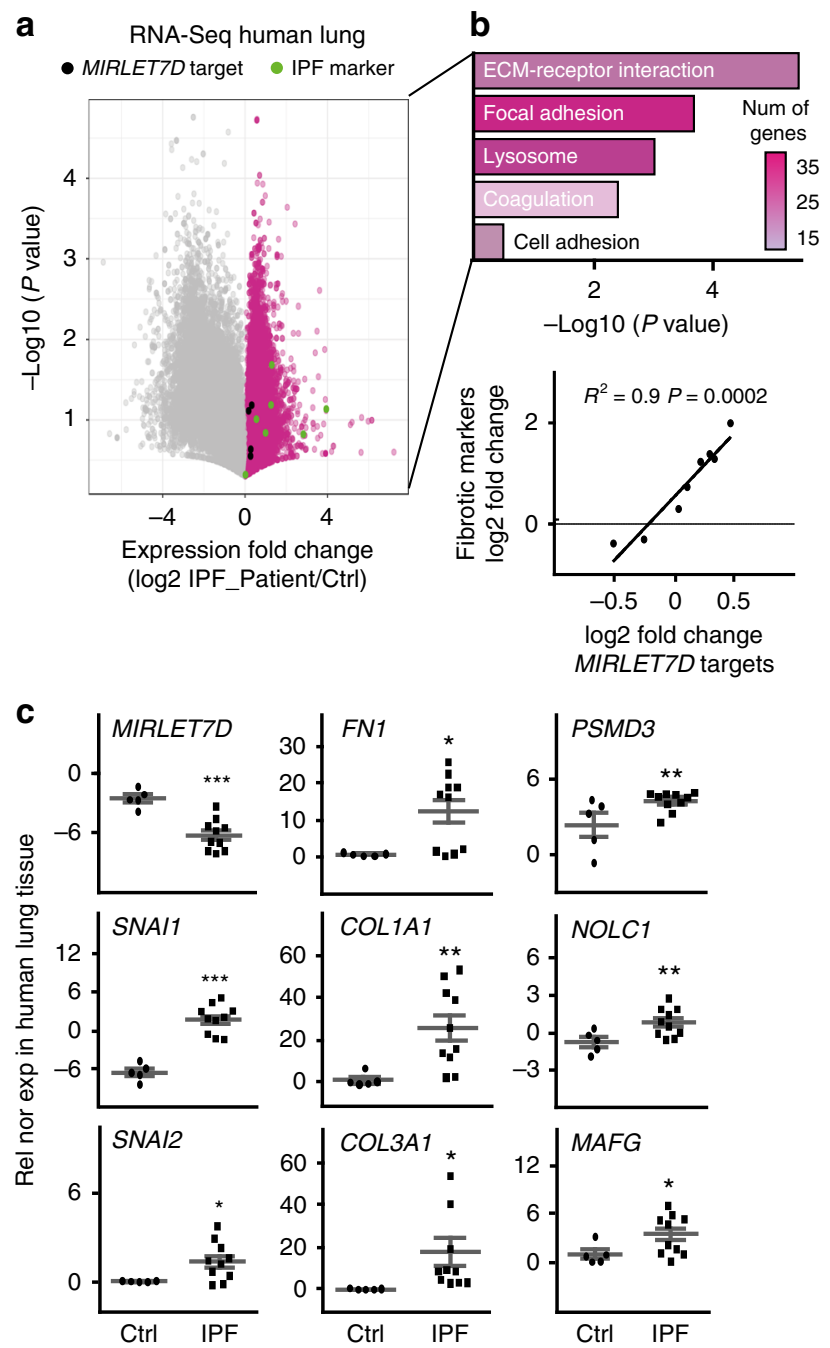

Fig. 1 Nuclear MIRLET7D targets can be used as novel IPF markers. a RNAsequencing in lung homogenates from $\mathrm{Ctrl}$ and IPF patients ${ }^{31}$. Volcano plot representing the significance $(-\log 10 P$-values after Welch's $t$-test) vs. expression fold change (log2 expression ratios) between average of $\mathrm{Ctrl}$ and two IPF patients. Magenta dots show transcripts with positive log2 expression ratios. Black dots show MIRLET7D targets. Green dots show fibrotic markers. b Top: KEGG-based enrichment analysis of transcripts upregulated in both IPF patients (magenta dots in a) using DAVID bioinformatics tool and plotted by highest significance $(-\log 10$ of modified Fisher's exact $P$-value). Color of the bar represents number of genes in each group based on the legend. ECM, extracellular matrix. Bottom: correlation analysis between MIRLET7D targets and fibrotic by linear regression of log2 FC value of a single MIRLET7D target paired with a single fibrotic marker from the two selected patients. All values were patient-matched and correlation clustering (data mining) from negative to positive values. c Mature MIRLET7D-specific TaqMan assay and qRT-PCR analysis of the indicated coding RNAs (cRNA) using total lung homogenates from $\mathrm{Ctrl}$ ( $n$ $=5$ biologically independent samples) and IPF patients ( $n=10$ biologically independent samples). Data are shown as means \pm SEM. Asterisks in all plots, $P$-values after unpaired $t$-test, one-tailed, ${ }^{\star \star \star} P<0.001 ;{ }^{\star \star} P<0.01 ;{ }^{\star} P$ $<0.05$. Source data are provided as a Source Data file

expression of ncRNAs associated to MIRLET7D targets in IPF fibroblasts. Our RNA-seq in human primary fibroblasts was confirmed by expression analysis of representative MIRLET7D targets by qRT-PCR (Fig. 2b). Furthermore, promoter analysis of the same MIRLET7D targets by chromatin immunoprecipitation (ChIP; Fig. 2c) showed decreased levels of various subunits of the
RNA exosome complex (EXOSC10, EXOSC5, and EXOSC1), the heterochromatin mark H3K27me3 (trimethylated Lys-27 of histone 3), and the enzyme mediating this histone modification (EZH2), whereas the levels of transcription initiating S5 phosphorylated RNA polymerase II (POLII) increased in IPF, compared with Ctrl fibroblasts.

To further characterize the human MiCEE complex, we performed western blotting (WB) analysis of proteins after miRNA pulldown (miR-Pd) using the nuclear fraction of human Ctrl lung fibroblasts and biotinylated control miRNA (MIRCTRL) or MIRLET7D as bait (Fig. 3a). Several components of the MiCEE complex, including C1D, EXOSC10, EXOSC1, and EZH2, were specifically enriched after MIRLET7D-Pd. Notably, these results were confirmed by TaqMan-based miRNA enrichment analysis after chromatin RNA IP (Ch-RIP) using antibodies specific for different subunits of the MiCEE complex (Fig. 3b). All of the MiCEE subunits tested specifically bound mature MIRLET7D in Ctrl fibroblasts. In addition, MIRLET7D binding by MiCEE components decreased in IPF fibroblasts. To link these results to MIRLET7D targets, we analyzed them by miR-Pd using the nuclear fraction of Ctrl or IPF primary fibroblasts transfected with biotinylated MIRCTRL or MIRLET7D (Fig. 3c) and found that MIRLET7D exclusively pulled down ncRNAs and not mRNAs. These results confirmed the specific interaction of MIRLET7D with ncRNAs but not with mRNAs. Consistent with these results, in silico analysis of ncRNAs related to MIRLET7D targets identified MIRLET7D-binding sites with relatively favorable minimum free energy $(\leq-20 \mathrm{kcal} / \mathrm{mol}$; Fig. $3 \mathrm{~d})$. These results provide a plausible explanation for the increased ncRNA and mRNA levels observed in IPF fibroblasts. Although MIRLET7D-targeted and exosome-mediated degradation is required to reduce the levels of ncRNAs, transcriptional silencing after heterochromatin formation is required for the reduction of the corresponding mRNAs. Our results indicate that the MiCEE complex mediates epigenetic silencing in humans as it does in mice ${ }^{25}$ and its function is compromised in IPF due to reduced MIRLET7D levels.

Our results indicate that the MiCEE complex is required for proper function of human lung fibroblasts. To test this hypothesis, we monitored the effects of loss-of-function (LOF) of different MiCEE components on fibrosis hallmarks (Fig. 4). LOF of MIRLET7D (by antagomiR probes) ${ }^{32}$, EXOSC10 (by short hairpin RNA construct, shRNA) ${ }^{25}$, and EZH2 (by small-molecule inhibitor, UNC-1999) ${ }^{35}$ in Ctrl human primary lung fibroblasts increased cell migration (Fig. 4a, b and Supplementary Fig. 3a, b), cell proliferation (Fig. 4c), and levels of fibrotic markers (Fig. 4d) to similar levels as in IPF fibroblasts, thereby demonstrating the requirement of the MiCEE complex for appropriate lung fibroblast functions.

Nuclear HDAC activity is reduced in IPF. Similar to MiCEE, $\mathrm{HDAC} 1$ and $\mathrm{HDAC} 2$ are commonly linked to heterochromatin formation $^{26}$. In addition, increased levels of HDACs have been shown in human lung tissue from IPF patients ${ }^{28-30}$. Thus, we decided to analyze the levels and the enzymatic activity of HDACs in protein extracts from patient-derived Ctrl and IPF lung fibroblasts. WB analysis of total protein extracts showed increased levels of HDAC1 and 2 in IPF as compared with Ctrl fibroblasts (Fig. 5a), correlating with previous reports ${ }^{28-30}$. However, two types of HDAC activity assays (Fig. 5b and Supplementary Fig. 4a) using nuclear and cytosolic extracts from Ctrl or IPF fibroblasts detected reduced HDAC activity in the nucleus of IPF fibroblasts when compared with Ctrl fibroblasts. Interestingly, MIRLET7D gain-of-function (GOF) in IPF fibroblasts reconstituted nuclear HDAC activity. On the other hand, 
a

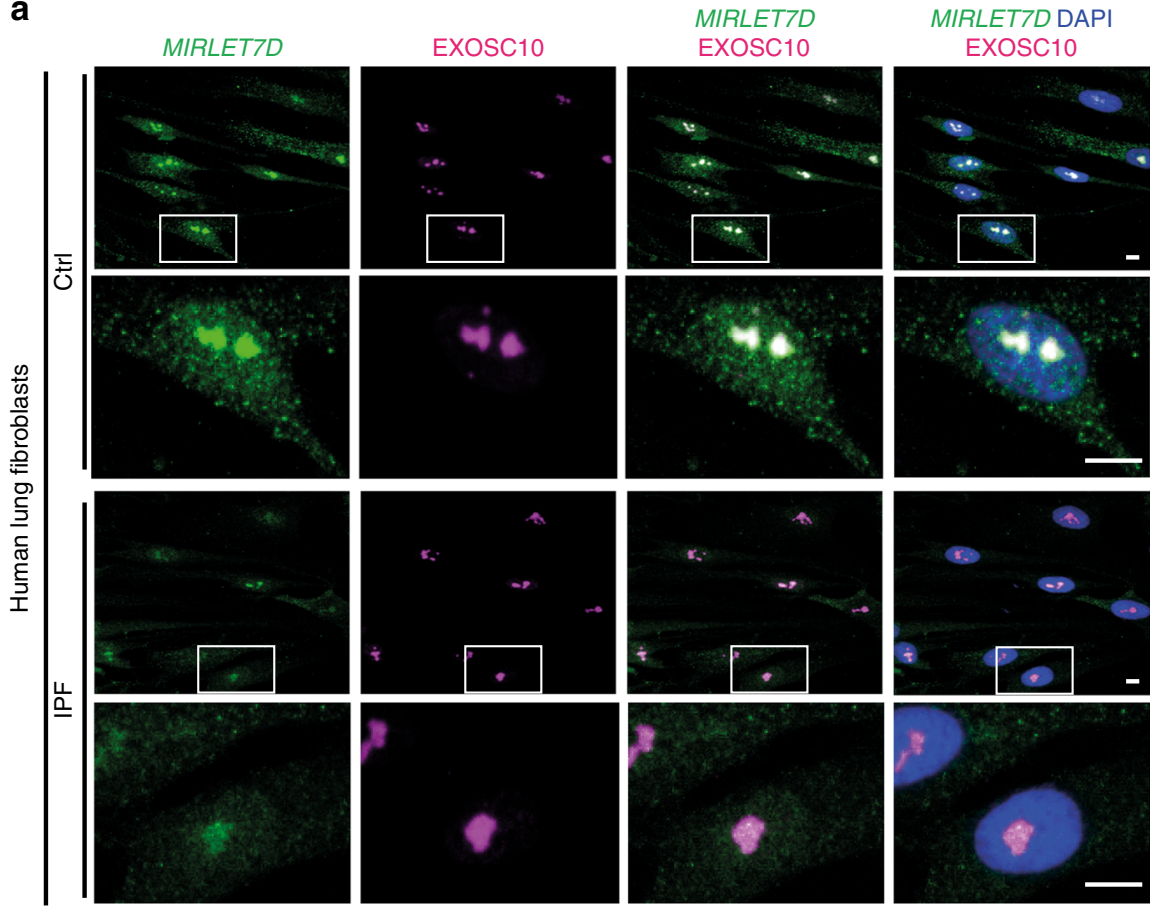

b

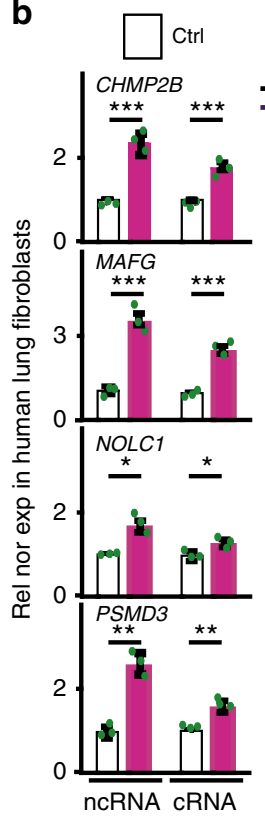
C IPF

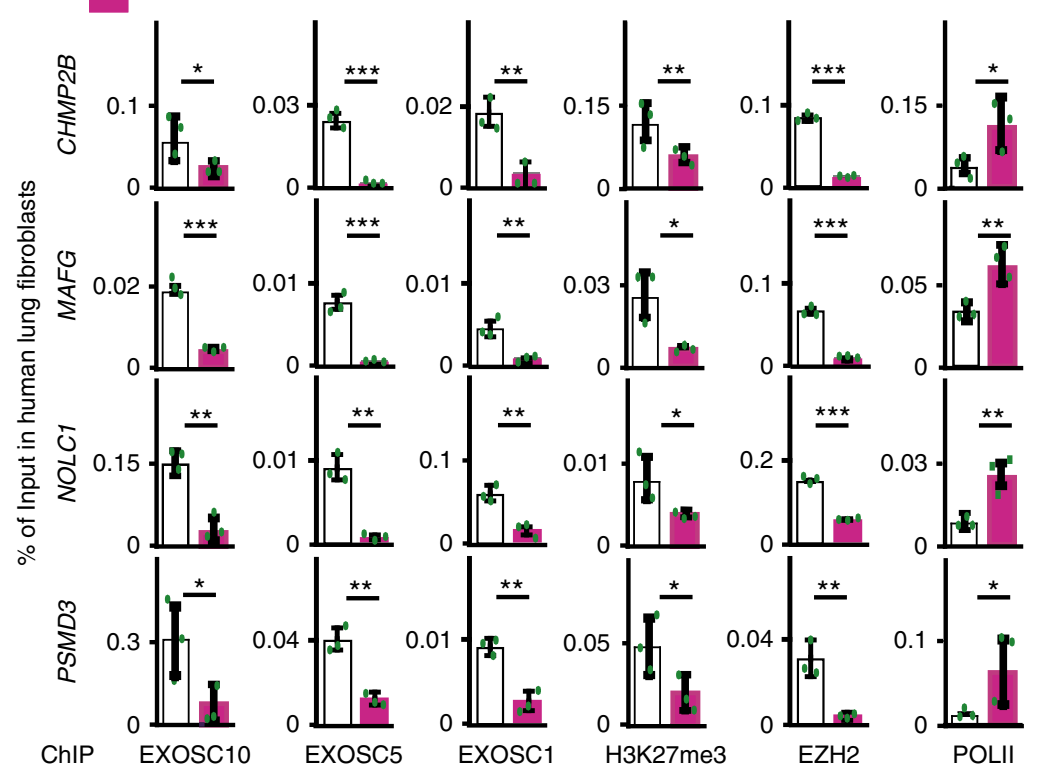

Fig. 2 Reduced MIRLET7D in IPF compromises MiCEE complex function. a Confocal microscopy after MIRLET7D-FISH and EXOSC10 immunostaining shows nuclear colocalization of MIRLET7D and EXOSC10 in Ctrl primary human lung fibroblasts and reduced level of MIRLET7D in IPF primary human lung fibroblasts. DAPI, nucleus. Scale bars, $5 \mu \mathrm{m}$. Squares are respectively shown below at higher magnification. $\mathbf{b}$ qRT-PCR-based analysis of noncoding RNA (ncRNA) and cRNA of representative nuclear MIRLET7D targets ${ }^{25}$ in Ctrl or IPF primary lung fibroblasts. See Supplementary Figs. 1 and 2. c ChIP analysis of promoters from representative MIRLET7D targets using the indicated antibodies and chromatin isolated from Ctrl or IPF primary human lung fibroblasts. In all bar plots, data are shown as mean (SD) ( $n=3$ biologically independent experiments). Asterisks in all plots, $P$-values after unpaired $t$-test, two-tailed, ${ }^{\star \star \star} P<0.001 ;{ }^{\star \star} P<0.01 ;{ }^{\star} P<0.05$; ns, not significant. Source data are provided as a Source Data file

cytosolic HDAC activity was higher in IPF fibroblasts compared with Ctrl fibroblasts. These results together indicated that HDAC1 and 2, although present in IPF nuclear extracts, might be inactive. To prove this hypothesis, nuclear extracts from Ctrl and IPF primary fibroblasts were fractionated by sucrose gradient ultracentrifugation (SGU) (Fig. 5c). Analysis of the obtained fractions by $\mathrm{WB}$ and TaqMan assay (top and middle) revealed that EXOSC10, EZH2, C1D, and MIRLET7D co-sedimented in fraction 5 of Ctrl nuclear extracts, supporting proper formation of the MiCEE complex. Interestingly, in IPF nuclear extracts the levels of MIRLET7D and C1D were reduced, and EXOSC10 and EZH2 sedimented in different fractions, suggesting a disruption of this complex. In addition, we analyzed SGU fractions by WB using HDAC1- and HDAC2-specific antibodies (bottom) and detected both deacetylases in the same fraction as MiCEE in Ctrl nuclear extracts. In contrast, HDAC1 and HDAC2 were detected 
a
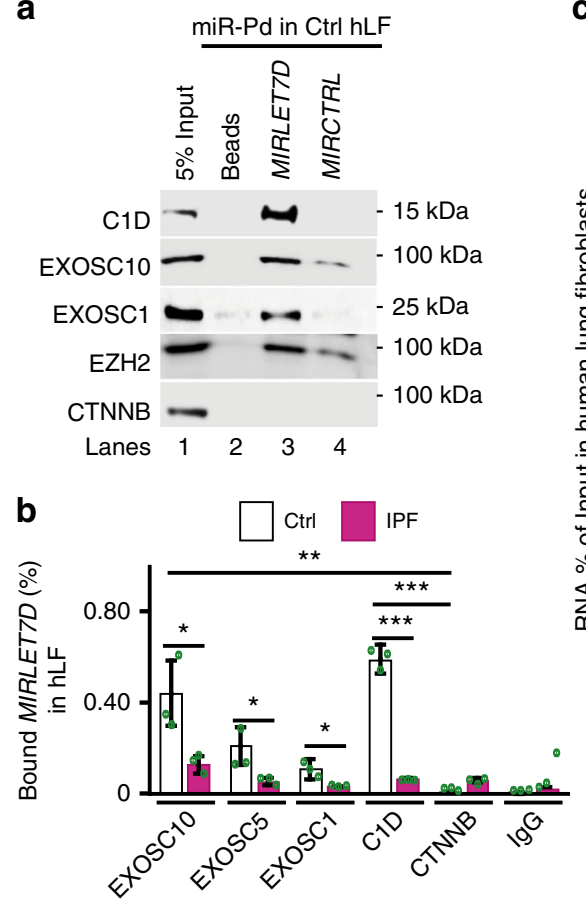

c

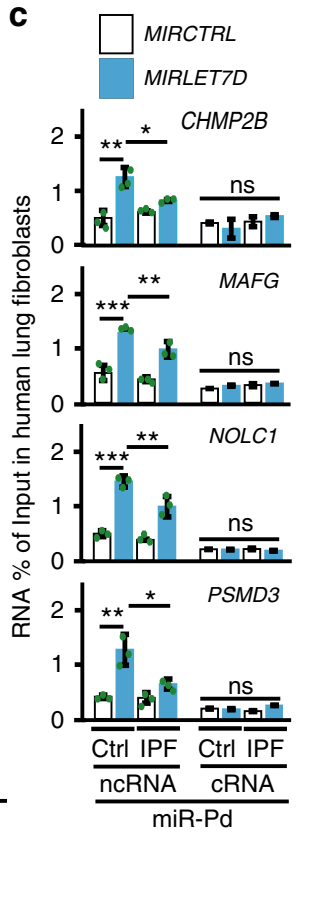

d

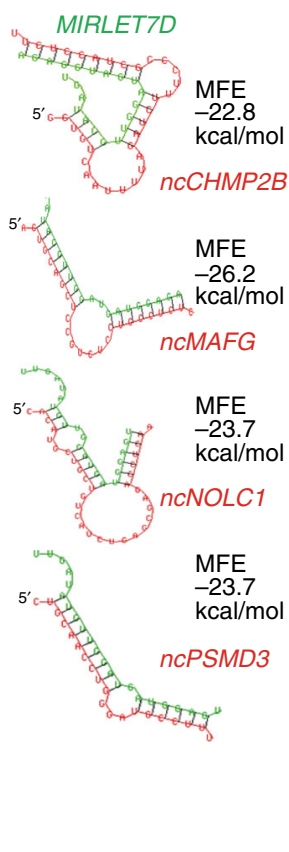

Fig. 3 MIRLETTD binds the MiCEE complex and ncRNAs from MIRLET7D targets. a Western blotting (WB) of precipitated proteins after miRNA pulldown (miR-Pd) using nuclear fraction from Ctrl primary human lung fibroblasts (hLF) and biotinylated MIRCTRL or MIRLET7D. Input, 5\% of starting material. Beads, control without probe. b Mature MIRLET7D-specific TaqMan assay after chromatin RNA immunoprecipitation (Ch-RIP) using the indicated antibodies and chromatin isolated from Ctrl or IPF hLF. CTNNB (beta-catenin) and IgG were used as negative controls. c qRT-PCR-based analysis of the indicated ncRNAs and cRNAs after miR-Pd from Ctrl or IPF hLF transfected with biotinylated MIRCTRL or MIRLET7D. d In silico analysis of ncRNAs from MIRLET7D target genes (magenta) identified MIRLET7D (green)-binding sites. MFE, minimum free energy. In all bar plots, data are shown as mean (SD) ( $n$ $=3$ biologically independent experiments). Asterisks in all plots, $P$-values after unpaired $t$-test, two tailed, ${ }^{\star \star \star} P<0.001 ;{ }^{\star \star} P<0.01 ;{ }^{\star} P<0.05 ;$ ns, not significant. Source data are provided as a Source Data file

in IPF nuclear extracts mainly in fractions 7 to 9 . Further analysis of SGU fractions by HDAC activity assays showed enrichment of deacetylase activity in fraction 5 of Ctrl nuclear extracts, whereas reduced HDAC activity was observed in IPF nuclear extracts. Strikingly, MIRLET7D transfection into IPF fibroblasts (Fig. 5c, right) was sufficient to reconstitute the MiCEE complex and the HDAC activity almost to similar levels as in Ctrl fibroblasts. The reconstituting effect of MIRLET7D-GOF on the MiCEE complex was confirmed by Co-IP of nuclear protein extracts from IPF lung fibroblasts using C1D-specific antibodies (Supplementary Fig. 4e). To further confirm the reduction of nuclear HDAC activity in IPF, we analyzed fractions containing HDAC1 and HDAC2 by IP assays of endogenous proteins using antibodies specific for panacetylated lysine (AcK) or phosphorylated serine $(\mathrm{PhS})$ and subsequent WB analysis of precipitated proteins using HDAC1or HDAC2-specific antibodies (Fig. 5d). It has been reported that HDAC1 acts upstream of HDAC2 and inactivation of HDAC1 leads to inactivation of $\mathrm{HDAC}^{36}$. Acetylation of specific lysine residues (K218, K220, K432, K438, K439, and K441) inactivates $\mathrm{HDACl}^{37}$, whereas phosphorylation of specific serine residues (S394, S422, and S424) subsequently inactivates $\mathrm{HDAC}^{38}$. Correlating with our HDAC activity assays (Fig. 5b, c), we detected inactive (AcK) HDAC1 and (PhS) HDAC2 in fractions 8 and 9 of IPF nuclear extracts, but not in fractions 5 and 6 of Ctrl nuclear extracts. The presence of inactive HDAC1 in the nucleus of IPF fibroblasts was also confirmed by proximity ligation assays (PLAs, Supplementary Fig. 4b) using AcK- and HDAC1-specific antibodies. Our results together confirmed that HDAC1 and HDAC2 are inactive in the nucleus of IPF fibroblasts. To further determine the role of HDAC1 activity, we transfected Ctrl primary fibroblasts with a constitutively inactive HDAC1 mutant
(inaHDAC1), in which specific lysine residues were mutated to glutamine (K218Q, K220Q, K432Q, K438Q, K439Q, and K441Q), mimicking $\mathrm{AcK}^{37}$. WB analysis of protein extracts from transfected Ctrl fibroblasts (Fig. 5e, lanes 1-2) showed increased levels of FN1, COL1A1, and ACTA1 after overexpression of inaHDAC1. The levels of these fibrosis markers in transfected Ctrl fibroblasts were similar to non-transfected IPF fibroblasts (compare lanes 2 and 3). Interestingly, transfection of IPF fibroblasts with a hyperactive HDAC1 mutant (actHDAC1), in which specific lysine residues were mutated to arginine (K218R, K220R, K432R, K438R, K439R, and K441R) to avoid $\mathrm{AcK}^{37}$, reduced the levels of the fibrosis markers (lanes 3-4) to levels similar to nontransfected Ctrl fibroblasts (compare lanes 4 and 1). To link the results obtained using protein extracts from primary fibroblasts with the MIRLET7D target genes, we analyzed these genes by ChIP, ChIP-reChIP (ChIP and sequential ChIP) (Supplementary Fig. 4c, d and Supplementary Discussion). These results demonstrated accumulation of inactive HDAC1 and HDAC2 at the promoters of MIRLET7D targets in IPF fibroblasts (Supplementary Fig. 4c, d). All these results together indicate that reduced nuclear HDAC activity in IPF interferes with proper MiCEE function and is crucial for fibrosis.

It has been previously suggested that removal of the acetyl group from $\mathrm{H} 3 \mathrm{~K} 27$ has to take place before the same amino acid residue can be methylated ${ }^{39}$. To confirm this sequential order of events in our experimental system, we analyzed the promoters of MIRLET7D targets by ChIP using antibodies specific for acetylated H3K27 (H3K27ac) or trimethylated H3K27 (H3K27me3) and chromatin isolated from IPF fibroblasts after MIRCTRL or MIRLET7D transfection alone or in combination with shRNAs specific for HDAC1 (shHDAC1; Supplementary 


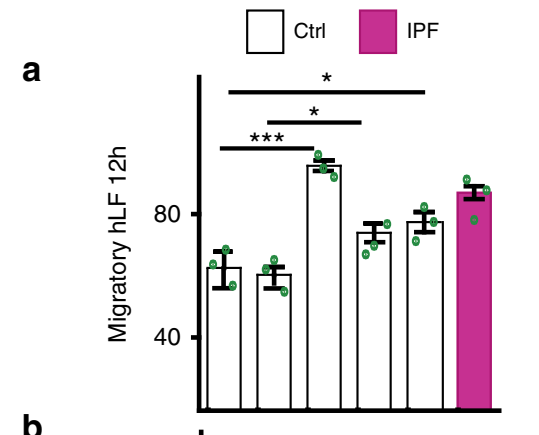

b

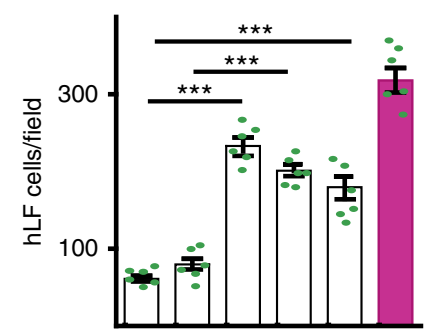

C

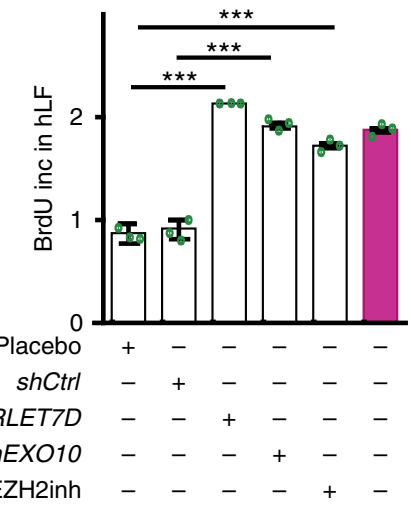

d

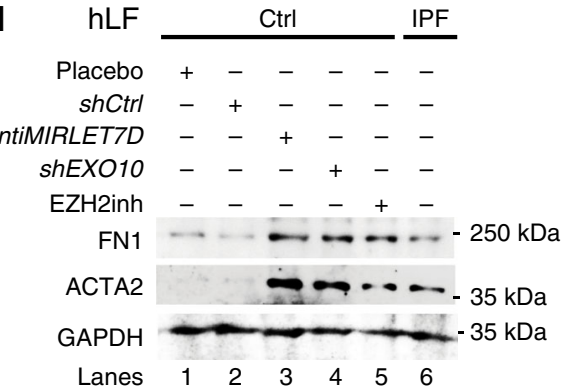

Fig. 4 Loss-of-function of MiCEE components increased fibrosis hallmarks. a-d LOF of MIRLET7D by an antagomir (antiMIRLET7D), EXOSC10 by a short hairpin RNA construct (shEXO10), and EZH2 by the smallmolecule inhibitor UNC-1999 (EZH2inh) in Ctrl human primary lung fibroblasts (hLF) increased cell migration (a, b), cell proliferation (c), and protein levels of fibrotic markers (d) to similar levels as in IPF hLF. Scratch migration assays (a) of Ctrl or IPF hLF quantified as the migratory distance $12 \mathrm{~h}$ after scratch (see Supplementary Fig. 3a). Quantification of Transwell invasion assays followed by hematoxylin and eosin (H\&E) stain (b) of Ctrl or IPF hLF (see Supplementary Fig. 3b). BrdU incorporation assays (c) using Ctrl or IPF hLF. WB of whole-cell protein extracts from Ctrl or IPF hLF using the indicated antibodies (d). In all bar plots, data are shown as mean (SD) ( $n=3$ biologically independent experiments for $\mathbf{a}$ and $\mathbf{c} ; n=6$ biologically independent experiments for $\mathbf{b}$ ). Asterisks in all plots, $P$-values after unpaired $t$-test, two tailed, ${ }^{\star \star \star} P<0.001 ;{ }^{\star \star} P<$ $0.01 ;{ }^{\star} P<0.05$; ns, not significant. Source data are provided as a Source Data file
Fig. 5a) or EZH2 (shEZH2; Supplementary Fig. 5b). Consistent with our previous results ${ }^{25}$, MIRLET7D-GOF reduced the levels of the euchromatin mark $\mathrm{H} 3 \mathrm{~K} 27 \mathrm{ac}$, whereas the levels of the heterochromatin mark $\mathrm{H} 3 \mathrm{~K} 27 \mathrm{me} 3$ increased. Interestingly, although shHDAC1 counteracted the effect of MIRLET7D on both chromatin marks, shEZH2 only counteracted the effect of MIRLET7D on H3K27me3. Our results support that HDAC1mediated deacetylation of $\mathrm{H} 3 \mathrm{~K} 27$ is required and anticipates $\mathrm{EZH} 2$-mediated methylation of the same amino acid residue, thereby confirming previously suggested models ${ }^{39}$. Furthermore, expression analysis of MIRLET7D targets by qRT-PCR (Supplementary Fig. 5c) correlated with the chromatin changes detected by ChIP analysis (Supplementary Fig. 5a, b).

Active EP300 inactivates HDAC1 in IPF. It is known that EP300 acetylates HDAC1, thereby inactivating its HDAC activity ${ }^{37}$. Interestingly, RNA-binding modulates EP300 acetyltransferase activity ${ }^{40}$. Thus, we monitored the levels of total EP300 by WB in protein extracts from Ctrl and IPF fibroblasts (Fig. 6a and Supplementary Fig. 6a) and detected a slight increase of EP300 in IPF compared with Ctrl fibroblasts. Strikingly, detection of active EP300 (actEP300) using antibodies specific for EP300 phosphorylated at serine $1834^{41}$ showed remarkably increased actEP300 levels in IPF fibroblasts compared with Ctrl. Our WB results were confirmed by confocal microscopy of primary fibroblasts after immunostaining using antibodies specific for actEP300 (Supplementary Fig. 6b). Further, we analyzed the promoters of MIRLET7D targets by ChIP using EP300- or actEP300-specific antibodies (Supplementary Fig. 6c, left). In MIRCTRL-transfected IPF fibroblasts, we detected increased levels of both total EP300 and actEP300 in all promoters analyzed when compared with MIRCTRL-transfected Ctrl fibroblasts. Interestingly, MIRLET7D-GOF in IPF fibroblasts did not significantly change the levels of total EP300, but reduced the levels of actEP300, suggesting inactivation of EP300 rather than its release from promoters. This observation was confirmed by WB of protein extracts from IPF fibroblasts after MIRLET7D-GOF (Supplementary Fig. 6a, right), as well as by detection of inactive EP300 (inaEP300) by ChIP of MIRLET7D targets using antibodies specific for phosphorylated EP300 at serine $89^{42}$ (Supplementary Fig. 6c, right). To demonstrate the causal involvement of actEP300 during the inactivation of HDAC1 in IPF fibroblasts, we analyzed HDAC activity in nuclear protein extracts from Ctrl and IPF fibroblasts (Fig. $6 \mathrm{~b}$ and Supplementary Fig. 6d). We detected reduced HDAC activity in the nucleus of IPF fibroblasts when compared with Ctrl fibroblasts, confirming our previous results (Fig. 5b, c). Interestingly, treatment of IPF fibroblasts with the EP300 inhibitor CBP30 ${ }^{43}$ (EP300inh; Supplementary Fig. 7a) reconstituted the HDAC activity to levels similar to Ctrl fibroblasts, supporting the causal involvement of actEP300 during the inactivation of HDAC1 in IPF. Further analysis of MIRLET7D targets by ChIP (Supplementary Fig. 7c), ChIP-reChIP (Fig. 6c), and qRT-PCR (Supplementary Fig. 7d) in Ctrl and IPF fibroblasts, in which two methods of EP300-LOF were used, EP300inh treatment or transfection of dominant-negative EP300 (dnEP300; Supplementary Fig. $7 \mathrm{a}, \mathrm{b})^{44}$, clearly demonstrated the causal involvement of actP300 during HDAC inactivation in the nucleus of IPF fibroblasts. These results together demonstrate a key role of active EP300 mediating inactivation of nuclear HDAC in IPF.

Analysis of SGU fractions (Fig. 5c) strongly suggested an interaction between HDAC1 and the MiCEE complex. This interpretation was confirmed by Co-IP assay using nuclear protein extracts from Ctrl primary fibroblasts (Fig. 6d). Endogenous HDAC1 co-immunoprecipitated C1D, EXOSC10, and 
a

$$
\frac{\text { Total protein from } \mathrm{hLF}}{\frac{\mathrm{Ctrl}}{123} \frac{\mathrm{IPF}}{123}}
$$

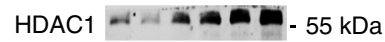

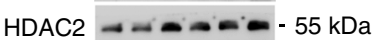

ACTA2 - $35 \mathrm{kDa}$

ACTB - - - - - $35 \mathrm{kDa}$

Lanes $\begin{array}{llllll}1 & 2 & 3 & 4 & 5 & 6\end{array}$ b

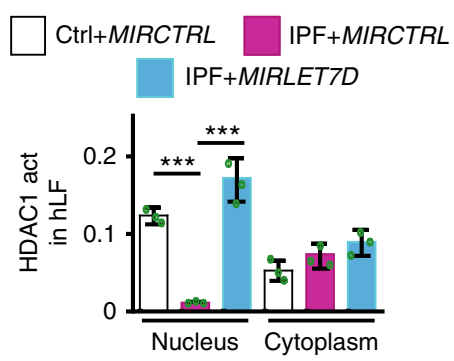

c

Nuclear protein from human lung fibroblasts

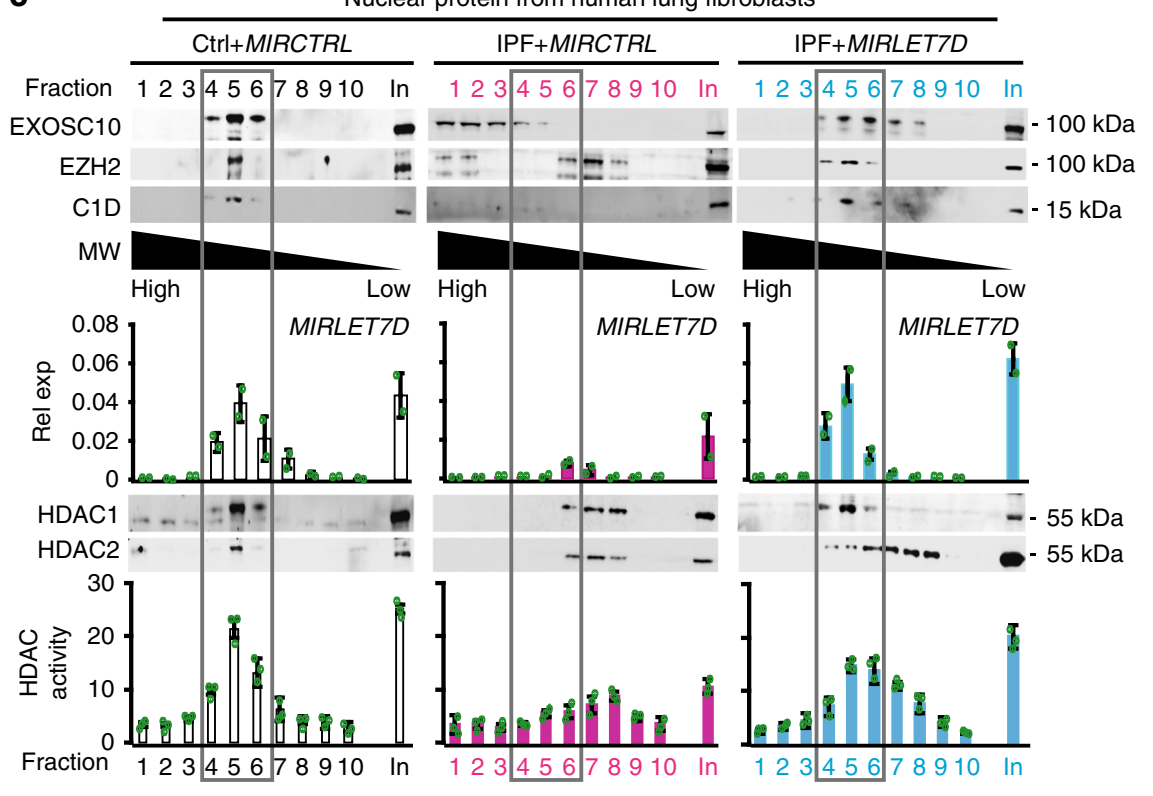

d

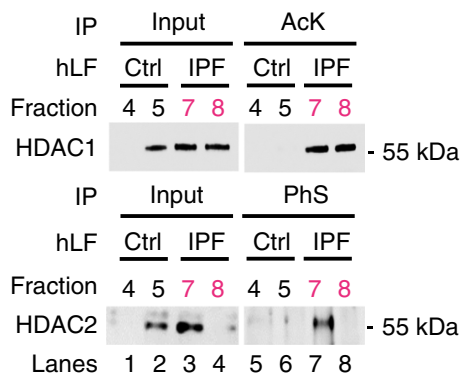

e

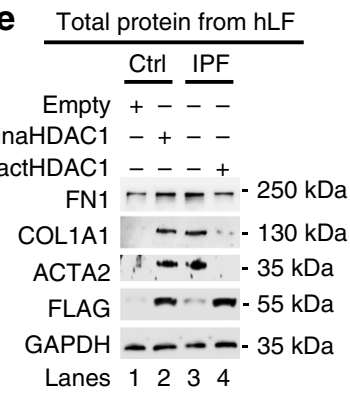

Fig. 5 Nuclear histone deacetylase activity is reduced in IPF. a Western blot (WB) using the indicated antibodies and whole-cell protein extracts from Ctrl and IPF primary human lung fibroblasts (hLF) derived from three different donors, each. $\mathbf{b}$ HDAC1-specific activity assay using nuclear or cytosolic protein extracts from Ctrl or IPF hLF that were transfected with control miRNA (MIRCTRL) or MIRLET7D probes. c WB using antibodies specific for MiCEE components (top) or HDAC1, or 2 (middle), MIRLET7D-specific TaqMan assay (middle), and fluorometric HDAC activity assay (bottom) of fractions after sucrose gradient ultracentrifugation (SGU) of nuclear extracts from Ctrl and IPF hLF transfected as in $\mathbf{b}$. Squares show fractions where MiCEE is expected. In, Input (5\% of material prior SGU); MW, molecular weight; Rel exp, relative expression. d WB using HDAC1 (top) or HDAC2 (bottom)-specific antibodies after immunoprecipitation using either pan-acetyl-lysine (AcK) or phospho-serine (PhS)-specific antibodies to precipitate endogenous inactive HDAC1 and HDAC2 from selected SGU fractions analyzed in c. Input, 5\% of IP starting material. e WB using the indicated antibodies and whole-cell protein extracts from Ctrl and IPF hLF that were transfected with an empty plasmid or expression constructs containing inactive (ina) or active (act) HDAC1 as indicated. In all bar plots, data are shown as mean (SD) ( $n=3$ biologically independent experiments for $\mathbf{a}$ and $\mathbf{c} ; n=2$ biologically independent experiments for $\mathbf{b}$ ). Asterisks in all plots, $P$-values after unpaired $t$-test, two tailed, ${ }^{\star \star \star} P<0.001 ;{ }^{\star \star} P<0.01 ;{ }^{\star} P<0.05$; ns, not significant. Source data are provided as a Source Data file. See Supplementary Fig. 4

EZH2, demonstrating the interaction with functional components of the MiCEE complex. To further investigate these interactions, we analyzed by Co-IP assay nuclear protein extracts from Ctrl fibroblasts that were transfected either with EXOSC10 full-length (fl) or a deletion mutant lacking the amino acids 1-285 (del3), thereby losing the ability to interact with $\mathrm{C}^{25} \mathrm{D}^{25}$ (Fig. 6e). Endogenous C1D co-immunoprecipitated HDAC1 in the presence of both, EXOSC10 $\mathrm{fl}$ and del3, supporting a direct C1D-HDAC1 interaction that does not require the previously described C1D-EXOSC10 interaction ${ }^{25}$. Interestingly, neither HDAC1 (Fig. 6d) nor C1D (Fig. 6e) immunoprecipitated EP300 in Ctrl fibroblasts. However, Co-IP assay using nuclear protein extracts from IPF fibroblasts (Fig. 6f) revealed that C1D interacts with active EP300, whereas C1D-HDAC1 interaction was 
a
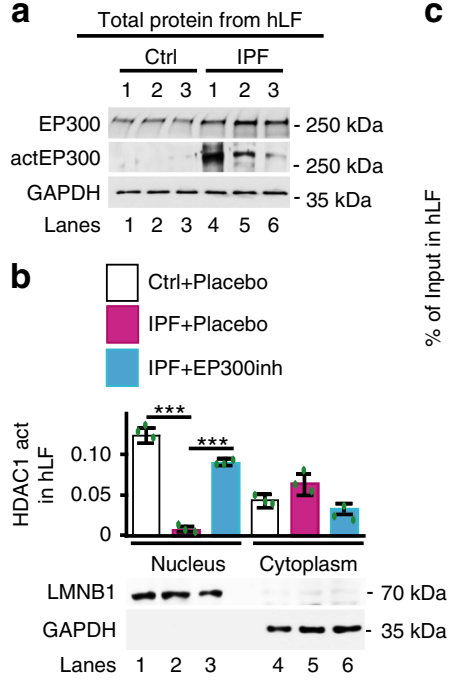

d

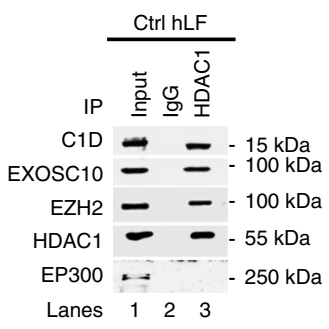

$\begin{array}{lllll}\text { C Ctrl+Placebo } & \text { Ctrl+EP300inh } & \text { Ctrl+dnEP300 } \\ \text { IPF+Placebo } & \text { IPF+EP300inh } & \text { IPF+dnEP300 }\end{array}$
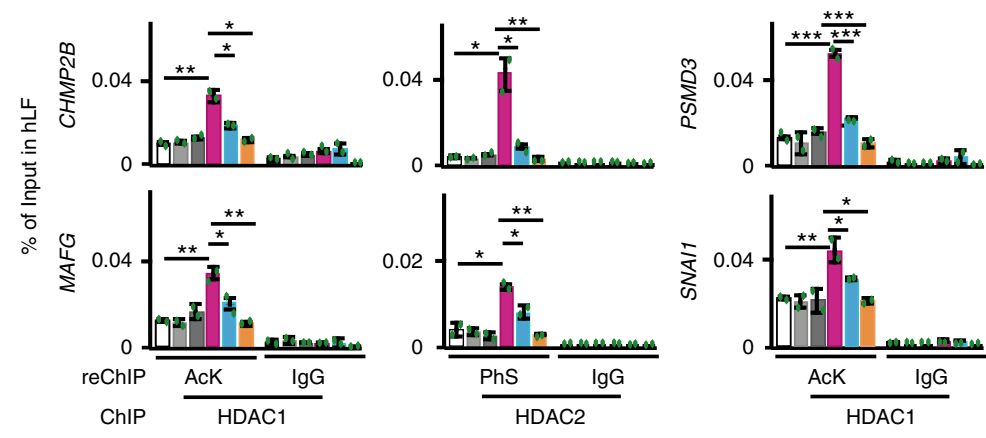
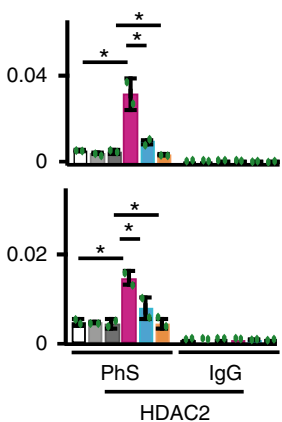

f
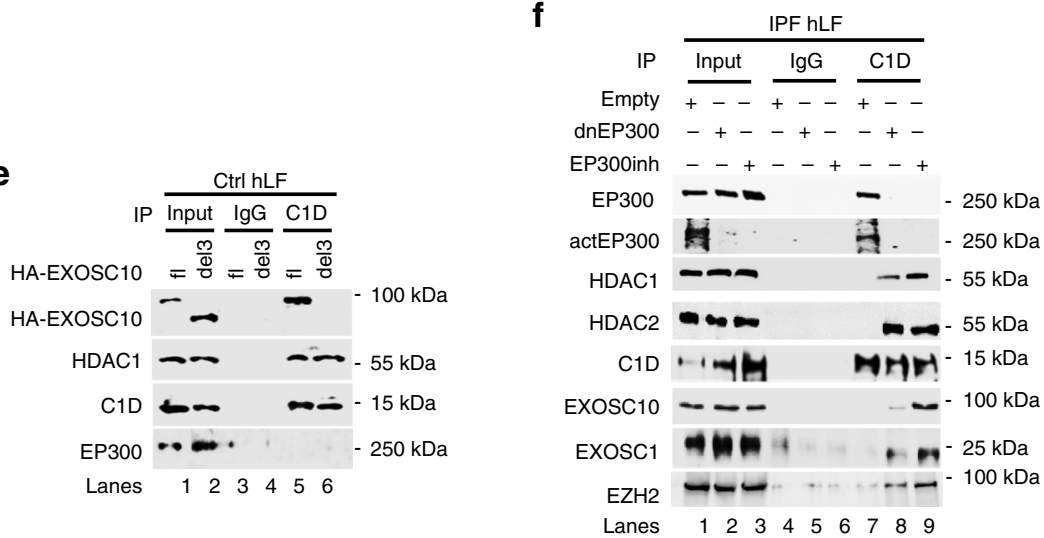

Fig. 6 Active EP300 inactivates HDAC1 in IPF. a WB using the indicated antibodies and whole-cell protein extracts from Ctrl and IPF lung fibroblasts (hLF) derived from three different donors each. ActEP300, active EP300. See Supplementary Fig. 6a-c. b Top: HDAC1-specific activity assay using nuclear or cytosolic protein extracts from Ctrl or IPF hLF treated with placebo (vehicle solution) or $10 \mu \mathrm{M} \mathrm{EP300} \mathrm{inhibitor} \mathrm{(EP300ihn,} \mathrm{CBP30).} \mathrm{Bottom:} \mathrm{WB}$ monitoring the quality of the fractions used in the HDAC1 activity assay. LMNB1, nuclear marker. GAPDH, cytosolic marker. See Supplementary Figs. 6d and 7a. c Sequential ChIP (ChIP-reChIP) analysis of representative MIRLET7D target promoters using HDAC1- or HDAC2-specific antibodies, followed by AcK- or PhS-specific antibodies, respectively, and chromatin isolated from Ctrl or IPF hLF after treatment with EP300inh or transfection of a dominantnegative EP300 (dnEP300) construct. IgG, immunoglobulin G (negative control). See Supplementary Fig. 7. d WB using the indicated antibodies after CoIP assay using immunoglobulin G (IgG; negative control) or HDAC1-specific antibodies to immunoprecipitate endogenous HDAC1 from nuclear protein extracts of Ctrl hLF. Input, 5\% of IP starting material. e WB after Co-IP assay as in $\mathbf{d}$ using C1D-specific antibodies to immunoprecipitate endogenous C1D from nuclear protein extracts of Ctrl hLF that were transfected with full-length (fl) or deletion mutant 3 (del3, lacking C1D interaction domain on amino acids 1-285) HA-tagged EXOSC10 constructs ${ }^{25}$. $\mathbf{f ~ W B}$ after Co-IP assay as in $\mathbf{d}$ using C1D-specific antibodies to immunoprecipitate endogenous C1D from nuclear protein extracts of IPF hLF that were either transfected with empty plasmid or with dnEP300 expression construct or treated with EP300inh as indicated. In all plots, data are shown as mean (SD) ( $n=3$ biologically independent experiments in $\mathbf{b} ; n=2$ biologically independent experiments in c); asterisks, $P$-values after unpaired $t$-test, two tailed, ${ }^{\star \star \star} P<0.001 ;{ }^{\star \star} P<0.01 ;{ }^{\star} P<0.05$. Source data are provided as a Source Data file

abolished (lane 7). Strikingly, two methods of EP300-LOF in IPF fibroblasts (EP300inh treatment and dnEP300 transfection; lanes 8 and 9) disrupted the C1D-EP300 interaction and reconstituted the C1D-HDAC1 interaction. Furthermore, the interaction of C1D with the other components of the MiCEE complex (EXOSC10, EXOSC1, and EZH2) was reconstituted after both methods EP300-LOF, supporting the appropriate formation of the MiCEE complex.

EP300inh counteracts fibrosis hallmarks in IPF models. Our results in primary fibroblasts and lung tissue support a key role of active EP300 in IPF. We therefore decided to determine the effect of EP300 inhibition on hallmarks of fibrosis in vivo (bleomycin mouse model, Fig. 7a-e and Supplementary Fig. 8a), ex vivo (PCLS, Supplementary Fig. 8b-d, Fig. 8d and 8f), and in vitro (human primary fibroblasts; Fig. $8 \mathrm{~b}-\mathrm{e}$ and $8 \mathrm{~g}$ ). In all these models of IPF, we detected upon EP300 inhibition reduced extracellular matrix protein deposition (Fig. 7b, c; Supplementary Fig. 8c, Fig. $8 \mathrm{a}$ and $8 \mathrm{~d}$ ), reduced levels of fibrotic markers, such as FN1,
COL1A1, and ACTA2 (Fig. 7d; Supplementary Fig. 8d and Fig. 8f), and reduced cell migration and proliferation (Fig. 8b, 8c and $8 \mathrm{e})$. All these results demonstrate the anti-fibrotic effects of EP300inh. Strikingly, transfection of constitutively inaHDAC1 mutant counteracted EP300inh (Fig. 8e and 8g), thereby supporting the causal involvement of active HDAC1 during the antifibrotic effects elicited by EP300 inhibition. Our results support a model in which HDAC1 interacts with the MiCEE complex via C1D in the nucleus of human Ctrl fibroblasts (Fig. 9a). In addition, we found that HDAC-mediated histone deacetylation is required and anticipates MiCEE-mediated heterochromatin formation. However, in the nucleus of human IPF fibroblasts (Fig. 9b), we detected both increase of active EP300, which inactivates HDAC1 and disrupts HDAC1-C1D interaction, and reduced levels of MIRLET7D, which compromises MiCEEmediated epigenetic gene silencing. Further dissecting the part of our model, in which reduced HDAC1 activity was detected in the nucleus of IPF fibroblasts (Fig. 5b, c), we also found that inaHDAC1 is sufficient to increase the levels of fibrotic markers in Ctrl fibroblasts (Fig. 5e). These results support a key role of 
a

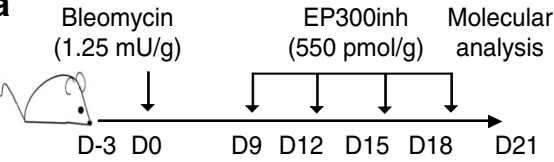

b
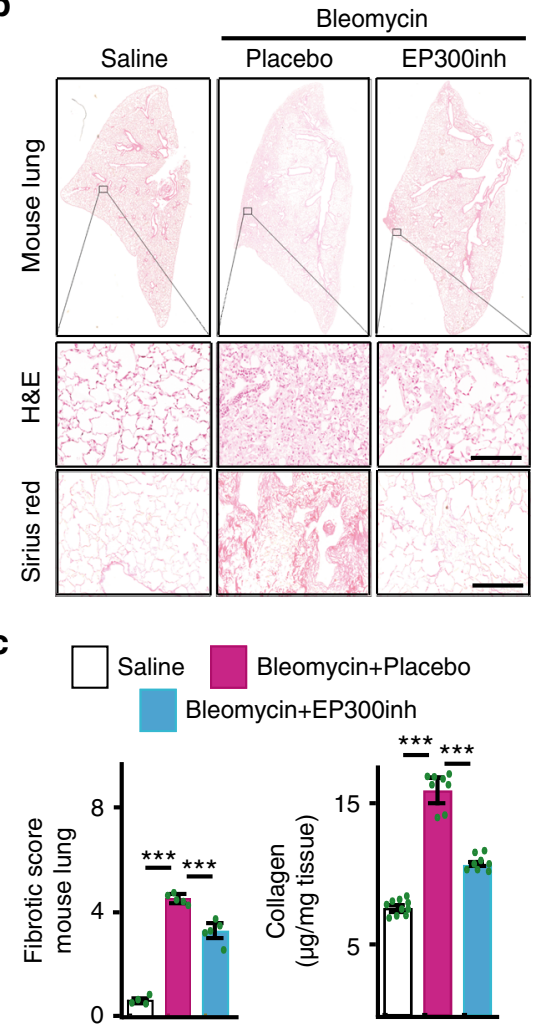

d

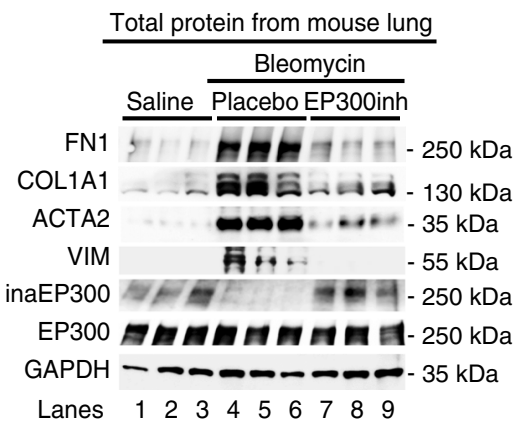

e

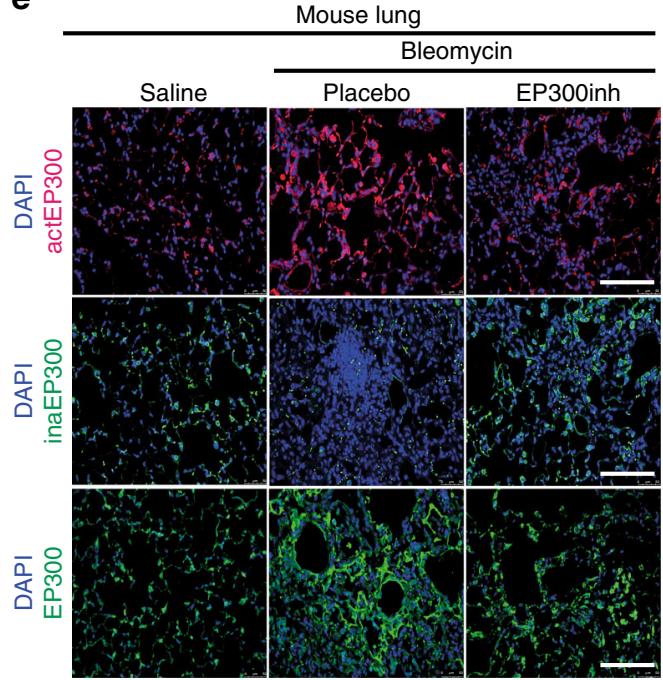

Fig. 7 EP300 inhibition counteracts fibrosis hallmarks in bleomycin mouse model. a Scheme shows experimental setup. Three group of mice (control group $n=4$, experimental groups $n=5$, in each group biologically independent animals) were orotracheally instilled with saline or bleomycin (1.25 mU per g body weight) at day 0 and subsequently orotracheally instilled with placebo (vehicle solution) or EP300 inhibitor (EP300inh, CBP30, 550 pmol per g body weight) on days 9, 12,15, and 18. Mice were collected for analysis at day 21. b Top: representative histological analysis using hematoxylin and eosin (H\&E) stain on left lung sections from mice treated as in a. Squares are shown below at higher magnification. Bottom, representative light microscopy after Sirius red staining monitoring collagen deposition on left lung sections from mice treated as in a. Scale bar $=100 \mu$ m. See Supplementary Fig. 8. c Left: fibrotic (Ashcroft) score quantification from representative Sirius red staining on left lung sections from mice treated as in a. Right, collagen content quantification by Sircol assay using lung homogenates from mice treated as in $\mathbf{a}$. Data are shown as mean (SD) ( $n=10,8$, or 7 biologically independent samples); asterisks, $P$-values after unpaired $t$-test, two tailed, ${ }^{\star \star \star} P<0.001$. Source data are provided as a Source Data file. $\mathbf{d}$ WB using the indicated antibodies and lung homogenates from three representative mice from each experimental group described in a. e Confocal microscopy after immunostaining using EP300, actP300, or inaEP300-specific antibodies in lung sections from representative mice from each experimental group described in a. ActEP300, active EP300; inaEP300, inactive EP300. DAPI, nuclear staining. Scale bar, $500 \mu \mathrm{m}$

HDAC inactivation during IPF. Interestingly, EP300-LOF experiments (Fig. 6b, c and Supplementary Fig. 7a-d) demonstrated the requirement of actP300 for HDAC inactivation in IPF fibroblasts. Moreover, overexpression of inaHDAC1 counteracted the anti-fibrotic effects of EP300inh (Fig. 8e and 8g). Our results clearly support the causal involvement of active EP300 in mediating the inactivation of nuclear HDAC1 during IPF.

\section{Discussion}

Previous reports have shown increased levels of HDACs in human lung tissue from IPF patients ${ }^{28-30}$, as we have detected in total protein extracts from IPF fibroblasts (Fig. 5a). However, these works also reported an increased HDAC activity in IPF. These apparent discrepancies could be explained by differences in the experimental systems. We have determined the enzymatic activity of HDACs in the nucleus, as well as HDAC1-specific enzymatic activity in the nucleus and cytosol of human primary fibroblasts from Ctrl and IPF patients, whereas the other reports neither discriminated between the cytosolic and nuclear fractions of cells used, nor directly determined the enzymatic activity stage of the HDACs. We detected reduced HDAC activity in the nucleus of IPF fibroblasts, whereas HDAC activity was increased in the cytosol of IPF fibroblasts (Fig. 5b, c and Supplementary Fig. $4 a)$. Our work has implications on the proposed use of HDAC inhibitors as potential treatment against pulmonary fibrosis ${ }^{28-30}$. Whereas inactivation of cytosolic HDAC might be favorable for IPF patients, inactivation of nuclear HDAC might be counterproductive. Consistently with our hypothesis, it has been reported that activation of the NAD +-dependent HDAC SIRT1 (Silent information regulator type 1) attenuated lung fibrosis ${ }^{45,46}$. Thus, a therapy against IPF based on EP300 inhibition might be more specific by targeting and reconstituting nuclear HDAC1 activity. Supporting this line of ideas, EP300 inhibition has been shown to ameliorate fibrosis in other organs ${ }^{47}$. Furthermore, inhibition of the EP300-related protein CBP (CREB-binding protein) reversed 
a

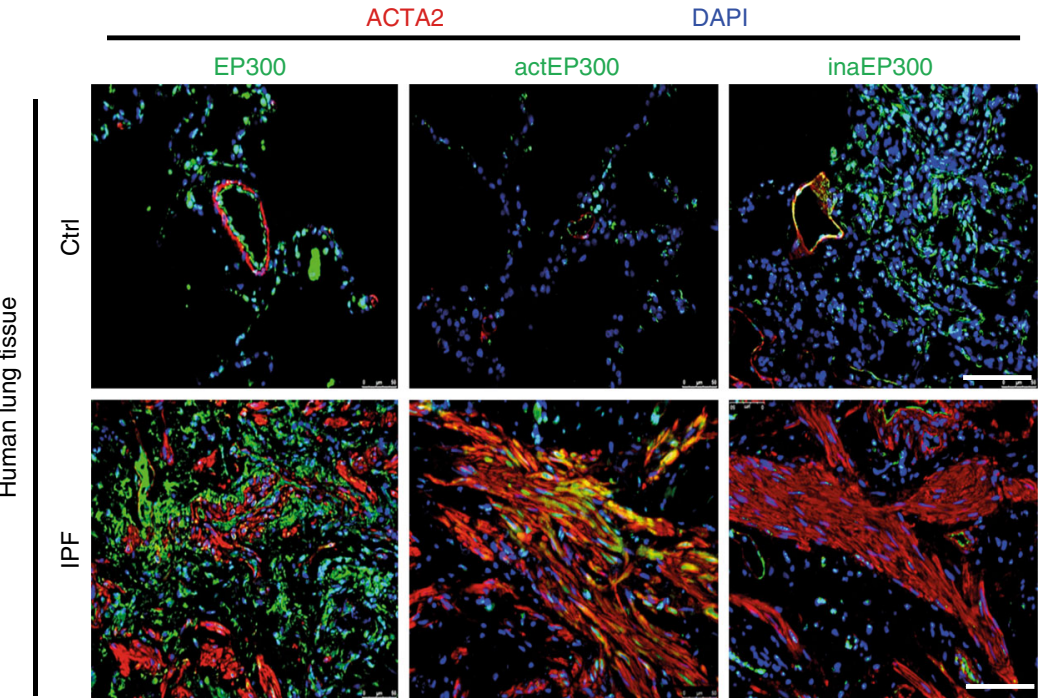

b
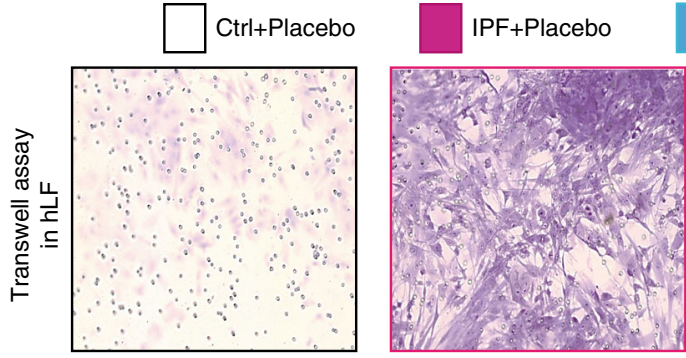

IPF+EP300inh

C

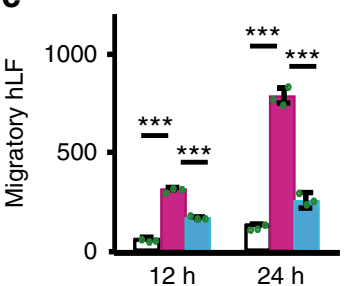

f

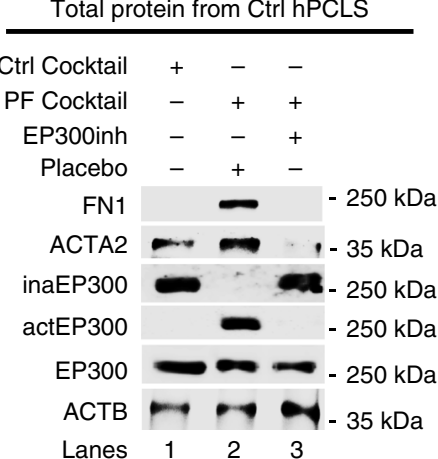

d

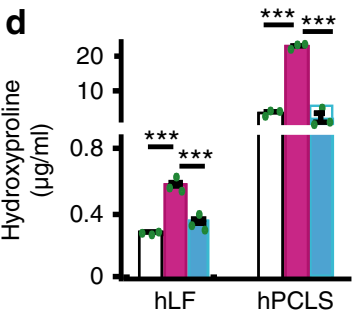

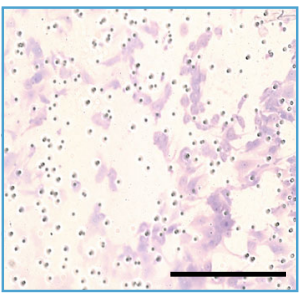

e

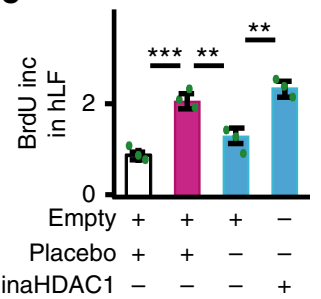

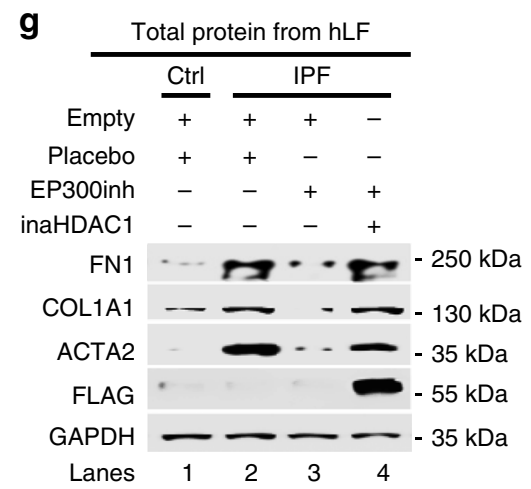

Fig. 8 EP300inh counteracts fibrosis hallmarks in IPF models. a Confocal microscopy after ACTA2, EP300, actEP300, and inaEP300 immunostaining in representative lung sections from Ctrl and IPF patients shows actEP300 increase in IPF. ActEP300, active EP300. DAPI, nucleus. Scale bars, $500 \mu$ m. b Transwell invasion assay followed by hematoxylin and eosin (H\&E) stain in human Ctrl or IPF lung fibroblasts (hLF) treated with placebo or EP300inh as indicated. Scale bar, $200 \mu \mathrm{m}$. c Scratch migration assay of Ctrl and IPF hLF treated as in b was quantified as the migratory distance 12 and $24 \mathrm{~h}$ after scratch. d Hydroxyproline assay for collagen content in hLF and human precision-cut lung slices (hPCLS) treated as in $\mathbf{b}$. e Proliferation assay by BrdU incorporation using Ctrl or IPF hLF treated with placebo or EP300inh and/or transfected with empty plasmid or inactive HDAC1 expression construct as indicated. $\mathbf{f}$ WB using the indicated antibodies and whole-cell protein extracts from hPCLS that were treated with Ctrl or pro-fibrotic (PF) cocktail and placebo or EP300inh as indicated. $\mathbf{g}$ WB using the indicated antibodies and whole-cell protein extracts from Ctrl or IPF hLF treated as in e. In all bar plots, data are shown as mean (SD) ( $n=3$ biologically independent experiments); asterisks, $P$-values after unpaired $t$-test, two tailed, ${ }^{\star \star \star} P<0.001$. Source data are provided as a Source Data file 

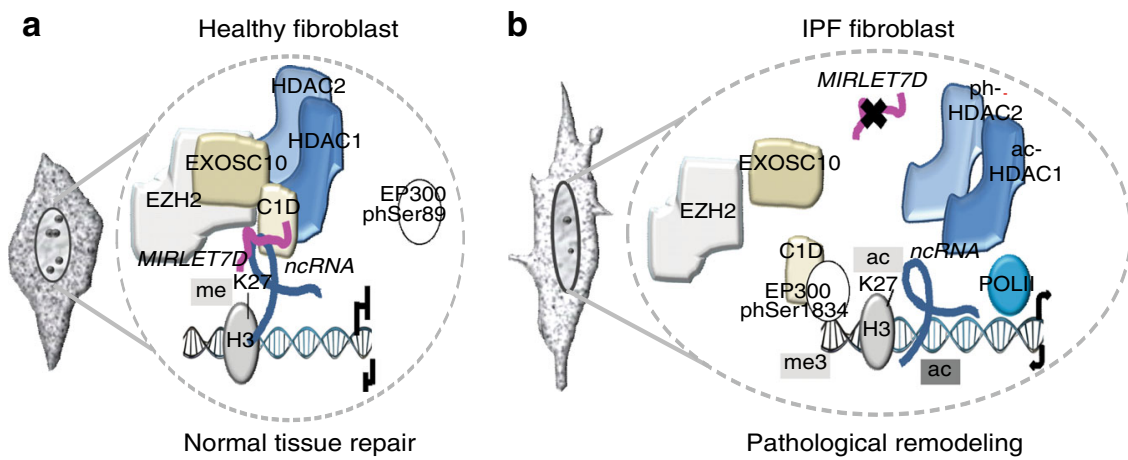

Fig. 9 Model. a In healthy lung fibroblasts, C1D-bound MIRLET7D-ncRNA duplexes attract active HDAC1/2 and the MiCEE complex to specific loci. Active HDAC1/2 deacetylate H3K27, whereas EZH2 mediates trimethylation of H3K27, thereby leading to heterochromatin formation and transcriptional silencing. $\mathbf{b}$ In IPF lung fibroblasts, reduced MIRLET7D and active EP300, which inactivates HDAC1/2 and interacts with C1D, disrupt the MiCEE complex interfering with heterochromatin formation and transcriptional silencing at specific loci

pulmonary fibrosis ${ }^{48}$. Chromatin is the physiological template for all DNA-dependent processes including transcription, replication, recombination, and repair ${ }^{49}$. However, the molecular mechanisms underlying the changes in chromatin structure mediating transcriptional regulation after EP300 inhibition in fibrosis was sparsely investigated prior to our study. The EP300inh used here is a potent small-molecule inhibitor of the bromodomaincontaining transcription factor EP300 ${ }^{50}$ (Supplementary Fig. 9). The results presented in this manuscript using EP300inh in vitro (Fig. 8b-e, 8g, Supplementary Fig. 7), in vivo (Fig. 7a-e), and ex vivo (Fig. 8d, 8f, and Supplementary Fig. 8) strongly suggest that inhibition of the bromodomain is crucial for the role of EP300 during IPF. In addition, the results obtained after overexpression of dnEP300, which is an EP300 mutant lacking acetyltransferase activity due to an inactivating D1399Y point mutation $^{44}$, complemented and confirmed the results obtained by EP300inh treatment. Moreover, we observed increased levels of acetylated H3 in IPF fibroblasts (Supplementary Fig. 5d), suggesting that $\mathrm{HDAC1}$ inactivation by acetylation might be just one of the effects caused by active EP300 during IPF progression. However, subsequent mechanistic analysis elucidating the function of EP300 during IPF initiation and progression will be the scope of future work.

IPF is a fatal interstitial lung disease for which no cure exists. Although two drugs have been approved for the treatment of IPF in several countries ${ }^{51,52}$, the prognosis of IPF patients remains poor. Thus, the search for drugs against IPF continues and several compounds have been approved for Phase II development for treatment against IPF $^{53}$. Remarkably, we demonstrated that EP300 inhibition reduced fibrotic hallmarks in vitro using primary fibroblasts from Ctrl and IPF patients, in vivo using the bleomycin mouse model, and ex vivo using PCLS. Our study lays the groundwork towards more efficient therapies targeting the causes of IPF using EP300 inhibition.

\section{Methods}

Study design. This study was performed according to the principles set out in the WMA Declaration of Helsinki; the underlying protocols were approved by the ethics committee of Medicine Faculty of the Justus Liebig University in Giessen, Germany (AZ.111/08-eurIPFreg) and the Hannover Medical School (number 2701-2015). In this line, all patient and control materials were obtained through the European IPF Registry (www.pulmonary-fibrosis.net), the UGMLC Giessen Biobank (member of the DZL Platform Biobanking), and the Biobank from the Institute for Pathology of the Hannover Medical School as part of the BREATH Research Network. All participants provided informed written consent in their respective center of enrollment. We used anonymized patient material. Patient characteristics are summarized in the Supplementary Fig. 1b.

Animal experiments were performed in accordance with German animal protection laws and were approved by the local governmental animal protection committee (Regierungspräsidium Darmstadt, Germany, Veterinärdezernat, approval number B2/1165). Eight- to 10 -week-old male mice were orotracheally instilled with $2.5 \mathrm{U} / \mathrm{kg}$ body weight of bleomycin (Medac, Germany) that was dissolved in saline. Control groups were instilled with saline. Bleomycin-instilled mice were orotracheally instilled with Placebo or EP300inh $(11 \mathrm{nmol} / 20 \mathrm{~g}$ body weight) on $\mathrm{d} 9, \mathrm{~d} 12$, d15 and d18.

Quantification and statistical analysis. Depending on the data, different tests were performed to determine the statistical significance of the results. The values of the statistical tests used in the different experiments can be found in the Statistical Summary (Source Data file). Further details of statistical analysis in different experiments are included in the figures and figure legends. Briefly, two biological replicates of RNA-seq were analyzed by deep sequencing. For the rest of the experiments presented here, samples were analyzed at least in triplicates and experiments were performed three times. Statistical analysis was performed using Excel Solver and Graph Prism (v.5). All data are represented as mean \pm SD. T-test was used to determine the levels of difference between the groups and $P$-values for significance. $P$-values after $T$-test, ${ }^{\star} P \leq 0.05 ;{ }^{*} P<0.01$, and ${ }^{\star * *} P<0.001$.

Cell culture, transfection, and treatment. Primary fibroblast from Ctrl and IPF patients were cultured in complete MCDB131 medium ( $8 \%$ fetal calf serum (FCS), $1 \%$ L-glutamine, penicillin $100 \mathrm{U} / \mathrm{ml}$, streptomycin $0.1 \mathrm{mg} / \mathrm{ml}$, epidermal growth factor $0.5 \mathrm{ng} / \mathrm{ml}$, basic fibroblast growth factor $2 \mathrm{ng} / \mathrm{ml}$, and insulin $5 \mu \mathrm{g} / \mathrm{ml}$ )) at 37 ${ }^{\circ} \mathrm{C}$ in $5 \% \mathrm{CO}_{2}$. Because of the concern that the phenotype of the cells is altered at higher passage, cells between passages 4 and 6 were utilized in the experiments described here, except cells for screening were used at earlier passages. During subculturing, cells were washed with $1 \times$ phosphate-buffered saline (PBS), trypsinized with $0.25 \%(\mathrm{w} / \mathrm{v})$ trypsin, and subcultivated at the ratio of $1: 5$ to $1: 10$. Cells were transfected with miRNA and/or plasmid DNA using Lipofectamine 2000 (Invitrogen) following the manufacturer's instructions, and collected $48 \mathrm{~h}$ later for further analysis. Biotinylated MIRCTRL (Exiqon \#166323), MIRLET7D (Ambion \#17010), AntiMiR MIRLET7D-5p (Ambion \#17000), and AntiMiR-negative control (Ambion \#17010) were transfected at $20 \mathrm{nM}$ final concentration. Cy3-labeled sictrl (Ambion \#17011) was used to evaluate miRNA transfection efficiency. shRNA plasmids shEXOSC10 (Sigma \#EHU082601), shHDAC1 (EHU025841), and shEZH2 (EHU073481) were used for loss-of-function in primary fibroblasts. Empty vectors were used as a negative control. For expression of dnEP300 we used pCMV $\beta$-p300.DY-myc, which was a gift from Tso-Pang Yao (Addgene plasmid \#30490; http://n2t.net/addgene:30490; RRID:Addgene_30490). Cells were treated with EZH2 inhibitor UNC-1999 (Sigma, \#SML0778) at $2 \mu \mathrm{M}$ final concentration for $24 \mathrm{~h}$. The solvent used for dissolving these inhibitors, following manufacturer's instruction, were used as a negative control.

Bacterial culture. For cloning experiments, chemically competent Escherichia coli TOP10 (ThermoFisher Scientific) were used for plasmid transformation. TOP10 strains were grown in Luria broth (LB) at $37^{\circ} \mathrm{C}$ with shaking at 180 r.p.m. for $16 \mathrm{~h}$ or on $\mathrm{LB}$ agar at $37^{\circ} \mathrm{C}$ overnight.

RNA isolation, RT, quantitative PCR, and TaqMan assay. Total RNA was isolated with Trizol (Invitrogen) and quantified using a Nanodrop Spectrophotometer (ThermoFisher Scientific, Germany). Synthesis of complementary DNA was performed using 1-2 $\mu \mathrm{g}$ total RNA and the High Capacity cDNA Reverse Transcription kit (Applied Biosystems). Quantitative real-time PCR reactions were performed using SYBR ${ }^{\circledast}$ Green on the Step One plus Real-time PCR system (Applied Biosystems). Housekeeping genes HPRT and GAPDH were used to normalize gene expression. Primer pairs used for gene expression analysis are 
described in Supplementary Table 1. The location of the ChIP primers is displayed as UCSC Genome Browser snapshots accordingly.

Quantitative TaqMan MicroRNA Assay (Applied Biosystems) was performed according to the manufacturer's instructions using primers and probes that specifically detect mature MIRLET7D (ThermoFisher Scientific \#4427975 ID 002283) and U6 control (ThermoFisher Scientific \#4426961 ID Hs03910430_s1).

miRNA pulldown. miR-Pd analysis was performed as described ${ }^{25}$ with minor adaptations. Ctrl and IPF primary fibroblasts were cultured in $10 \mathrm{~cm}$ dishes and transfected with Biotinylated hsa-MIRLET7D-5p-RNA (Exiqon \#279787) or Biotinylated MIRCTRL (Exiqon \#166320), at a final concentration of $20 \mathrm{nM}$ using Lipofectamine 2000 (Invitrogen) following the manufacturer's protocol. After $48 \mathrm{~h}$, cells were pelleted at $270 \mathrm{~g}$ for $5 \mathrm{~min}$. Cells were fixed with $1 \%$ glutaraldehyde (Sigma-Aldrich) to preserve RNA-Chromatin interactions. After washing three times with PBS, cell pellets were resuspended in $2 \mathrm{ml}$ hypotonic cell lysis buffer (10 $\mathrm{mM}$ Tris- $\mathrm{HCl}$ (pH 7.4), $1.5 \mathrm{mM} \mathrm{MgCl}_{2}, 10 \mathrm{mM} \mathrm{KCl}, 1 \mathrm{mM}$ dithiothreitol (DTT), $25 \mathrm{mM} \mathrm{NaF}, 0.5 \mathrm{mM} \mathrm{Na} \mathrm{VO}_{4}, 40 \mu \mathrm{g} / \mathrm{ml}$ phenylmethylsulfonyl fluoride, protease inhibitor (Calbiochem), and RNase inhibitor (Promega) in Diethyl pyrocarbonate (DEPC)-treated water) on ice for $10 \mathrm{~min}$ and then spun down at $700 \mathrm{~g}$ for $10 \mathrm{~min}$ at $4{ }^{\circ} \mathrm{C}$. Nuclear pellets were resuspended in $300 \mu \mathrm{l}$ nuclear lysis buffer $(50 \mathrm{mM}$ Tris$\mathrm{HCl}(\mathrm{pH} 7.4), 170 \mathrm{mM} \mathrm{NaCl}, 20 \%$ glycerol, $15 \mathrm{mM}$ EDTA, $0.1 \%$ (v/v) Triton X$100,0.2 \mathrm{mM}$ DTT, $20 \mathrm{mM} \mathrm{NaF}, 20 \mathrm{mM} \mathrm{Na}_{3} \mathrm{VO}_{4}, 40 \mu \mathrm{g} / \mathrm{ml}$ phenylmethylsulfonyl fluoride, protease inhibitor, and RNase inhibitor in DEPC-treated water). Nuclear fraction was sonicated (Bioruptor NextGen, Diagenode) at high amplitude for 10 cycles of $30 \mathrm{~s}$ (on/off) pulses. M280 streptavidin magnetic beads (ThermoFisher Scientific) were blocked for $1 \mathrm{~h}$ at $4^{\circ} \mathrm{C}$ in blocking buffer (10 mM Tris-HCl pH 6.5, $1 \mathrm{mM}$ EDTA, $1 \mathrm{mg} / \mathrm{ml}$ yeast tRNA, and $1 \mathrm{mg} / \mathrm{ml}$ bovine serum albumin (BSA)) and washed twice with $1 \mathrm{ml}$ washing buffer $(10 \mathrm{mM}$ Tris- $\mathrm{HCl} \mathrm{pH} 7.0,1 \mathrm{mM}$ EDTA, $0.5 \mathrm{M} \mathrm{NaCl}, 0.1 \%(\mathrm{v} / \mathrm{v})$ Triton X-100, RNAase inhibitor, and protease inhibitor). Beads were resuspended in $0.5 \mathrm{ml}$ washing buffer. The nuclear extract was then added to the beads and incubated for $1 \mathrm{~h}$ at $4{ }^{\circ} \mathrm{C}$ with slow rotation. The beads were then washed five times with $1 \mathrm{ml}$ washing buffer. RNA bound to the beads (pulled down RNA) and from 10\% of the extract (input RNA) was isolated using Trizol reagent LS (Invitrogen) after DNasel (NEB) treatment. The level of mRNA and ncRNA in the Biotinylated MIRLET7D-5p or Biotinylated MIRCTRL pulldown was quantified by qRT-PCR.

For miRNA-protein interaction analysis, nuclear protein extract after DNasel treatment incubated with $500 \mathrm{pMol}$ Biotinylated MIRLET7D-5p or Biotinylated MIRCTRL for $1 \mathrm{~h}$ with shaking at $4^{\circ} \mathrm{C}$. M280 streptavidin magnetic beads (ThermoFisher Scientific) were used for pulldown. Protein bound to the beads (miR-Pd) was incubated with $30 \mu \mathrm{l} 2 \times \mathrm{SDS}$ samples loading buffer, boiled at $95^{\circ} \mathrm{C}$ for $5 \mathrm{~min}$, spun down, and loaded on SDS-polyacrylamide gel electrophoresis (PAGE) for WB analysis.

Chromatin RNA immunoprecipitation. Ch-RIP analysis was performed as described ${ }^{54}$ with minor adaptations. Briefly, cells were crosslinked by $1 \%$ formaldehyde for $10 \mathrm{~min}$, lysed, and sonicated with Diagenode Bioruptor to disrupt and fragment genomic DNA. After centrifugation, the soluble chromatin was immunoprecipitated using antibodies specific against EXOSC10 (Abcam, \#50558, dilution 1:1000), C1D (Aviva, \#ARP50534_P050, dilution 1:200), EXOSC5 (Abcam, \#69699, dilution 1:500), EXOSC1 (Abcam, \#181167, dilution 1:1000), CTNNB (Abcam, \#7302, dilution 1:1000), and IgG as a control (Santa Cruz, \#sc2027). Precipitated chromatin complexes were removed from the beads by incubating with $50 \mu \mathrm{l}$ of $1 \%$ SDS with $0.1 \mathrm{M} \mathrm{NaHCO}_{3}$ for $15 \mathrm{~min}$, vortexing every 5 min, and followed by treatment with DNasel. Reverse crosslinked immunoprecipitated materials were used for RNA isolation by Trizol (Invitrogen). Isolated RNA was subjected to cDNA synthesis and further for TaqMan assay.

ChIP and sequential ChIP analysis. ChIP analysis was performed as described earlier ${ }^{17}$ with minor adaptations. Briefly, cells were crosslinked by $1 \%$ formaldehyde for $10 \mathrm{~min}$, lysed, and sonicated with Diagenode Bioruptor to an average DNA length of 500-600 bp. After centrifugation, the soluble chromatin was immunoprecipitated using specific antibodies anti-EXOSC10 (Abcam, \#ab50558), anti-EXOSC5 (Abcam, \#69699), anti-EXOSC1 (Abcam, \#181167), antiCTNNB (Abcam, \#7302), anti-EZH2 (Abcam, \#ab3748), anti-H3K27me3 (Millipore, \#07-449), anti-H3 (Abcam, \#1791), anti-H3K56ac (Epigentek, \#A4026-050), anti-H3K27ac (Abcam, \#ab4729), anti-HDAC1 (Abcam, \#ab7028), anti-HDAC2 (Abcam, \#ab16032), anti-POLII (Abcam, \#ab5408, anti-EP300 (Abcam, \#59240), anti-actEP300 (ThermoFisher, PA5-64531), anti-actEP300 (Biorbyt, \#34557), anti-inaEP300 (Santa Cruz, \#130210), anti-AcK (Cell Signaling, \#9441 S)), anti-PhS (Millipore, \#A-570-05-1000 × ), anti-IgG (Santa Cruz, \#sc2027), and anti-IgG (Santa Cruz, \#sc-2025). Precipitated chromatin complexes were removed from the beads by incubating with $50 \mu \mathrm{l}$ of $1 \% \mathrm{SDS}, 0.1 \mathrm{M} \mathrm{NaHCO}_{3}$, for $30 \mathrm{~min}$, while vortexing every $5 \mathrm{~min}$. Reverse crosslinked immunoprecipitated chromatin was subjected to quantitative PCR.

Sequential ChIP (ChIP-reChIP) was performed as described earlier ${ }^{17}$ with slight modifications. Briefly, re-ChIP was performed using specific antibodies antiHDAC1 (Abcam, \#ab7028), anti-HDAC2 (Abcam, \#ab16032), anti-AcK (Cell Signaling, \#9441 S)), anti-PhS (Millipore, \#A-570-05-1000×), anti-IgG (Santa Cruz, \#sc-2027), and anti-IgG (Santa Cruz, \#sc-2025). For re-ChIP experiments, complexes were eluted in $25 \mu \mathrm{l} 10 \mathrm{mM}$ DTT by incubation at $37^{\circ} \mathrm{C}$ for $30 \mathrm{~min}$. After centrifugation, the supernatant was diluted 20 times with re-ChIP buffer $(1 \%$ Triton X-100, $2 \mathrm{mM}$ EDTA, $150 \mathrm{mM} \mathrm{NaCl}, 20 \mathrm{mM}$ Tris-HCl pH 8.0) and subjected again to the ChIP procedure. Primer pairs used for gene promoter analysis are described in Supplementary Table 1.

Sucrose gradient ultracentrifugation. Sucrose gradients (5-40\%) were prepared in native nuclear lysis buffer ( $0.2 \%$ IPGAL, $100 \mathrm{mM} \mathrm{NaCl}, 50 \mathrm{mM}$ Tris-HCl pH 7.0, $1 \mathrm{mM}$ EDTA, $0.2 \mathrm{mM}$ DTT, $20 \mathrm{mM} \mathrm{NaF}, 20 \mathrm{mM} \mathrm{Na}_{3} \mathrm{VO}_{4}, 40 \mu \mathrm{g} / \mathrm{ml}$ phenylmethylsulfonyl fluoride, protease inhibitor, and RNase inhibitor in DEPCtreated water) in polyallomer centrifuge tube (Beckman). Nuclear Ctrl and IPF extracts were loaded in $5 \mathrm{ml} 5-40 \%$ sucrose gradients and centrifuged for $16 \mathrm{~h} 30$ $\mathrm{min}$ at $132,000 \times g$ in a SW50.1 ultracentrifuge rotor (Beckman). Following centrifugation, ten fractions $(500 \mu \mathrm{l}$ each) were collected manually from the bottom. Later, these fractions were used for WB analysis and RNA isolation using Trizol (Invitrogen) for TaqMan assay as described above.

Co-IP and WB analysis. Co-IP was performed as described ${ }^{17}$ with minor adaptations. A total of $5 \times 10^{7}$ cells were collected and washed three times in cold PBS, spun down at $270 \mathrm{~g}$ for $10 \mathrm{~min}$ at $4{ }^{\circ} \mathrm{C}$. Cells were incubated in $2 \mathrm{ml}$ of hypotonic cell lysis buffer $(10 \mathrm{mM}$ (pH 7.4) Tris- $\mathrm{HCl}, 1.5 \mathrm{mM} \mathrm{MgCl} 2,10 \mathrm{mM} \mathrm{KCl}, 1 \mathrm{mM}$ DTT, $25 \mathrm{mM} \mathrm{NaF}, 0.5 \mathrm{mM} \mathrm{Na} \mathrm{VO}_{4}, 40 \mu \mathrm{g} / \mathrm{ml}$ phenylmethylsulfonyl fluoride and protease inhibitor (Calbiochem)) on ice for $10 \mathrm{~min}$ and then spun down at $700 \mathrm{~g}$ for $10 \mathrm{~min}$ at $4{ }^{\circ} \mathrm{C}$. Nuclear pellets were resuspended in $300 \mu \mathrm{l}$ nuclear lysis buffer (50 mM Tris- $\mathrm{HCl}$ (pH 7.4), $170 \mathrm{mM} \mathrm{NaCl}, 20 \%$ glycerol, $15 \mathrm{mM} \mathrm{EDTA}, 0.1 \%$ (v/v) Triton X-100, $0.2 \mathrm{mM}$ DTT, $20 \mathrm{mM} \mathrm{NaF}, 20 \mathrm{mM} \mathrm{Na}_{3} \mathrm{VO}_{4}, 40 \mu \mathrm{g} / \mathrm{ml}$ phenylmethylsulfonyl fluoride and protease inhibitor). Pre-cleared nuclear protein lysates $(500 \mu \mathrm{g})$ were incubated with the appropriate antibodies on ice for $2 \mathrm{~h}$ and then $30 \mu \mathrm{l}$ protein-G-sepharose beads (GE Healthcare; equilibrated once in $10 \mathrm{ml}$ water and three times in washing buffer) were added and incubated overnight at $4^{\circ}$ C. Beads were collected and washed five times with $500 \mu \mathrm{l}$ ice-cold washing buffer. Thirty microliters of $2 \times$ SDS sample loading buffer was added to beads, boiled at $95^{\circ} \mathrm{C}$ for $5 \mathrm{~min}$, spun down, and loaded on SDS-PAGE for WB analysis. IP and WB analyses were performed using specific antibodies anti-EXOSC10 (Abcam, \#ab50558, dilution 1:1000), anti-EXOSC5 (Abcam, \#69699, dilution 1:500), antiEXOSC1 (Abcam, \#181167, dilution 1:1000), anti-CTNNB (Abcam, \#7302, dilution 1:1000), anti-EZH2 (Abcam, \#ab3748, dilution 1:1000), anti-SUV39H1 (Active Motif, \#39785, dilution 1:500), anti-H3 (Abcam, \#1791, dilution 1:1000), antiH3Ac (Abcam, \#ab47915, dilution 1:1000), anti-C1D (Aviva, \#ARP50534_P050, dilution 1:200), anti-HDAC1 (Abcam, \#ab7028, dilution 1:1000), anti-HDAC2 (Abcam, \#ab16032, dilution 1:1000), anti-FLAG M2 (Sigma, \#F1804, dilution 1:1000), anti-FN1 (Millipore, \#AB2033, dilution 1:1000), anti-COL1A1 (Sigma, \#C2456, dilution 1:500), anti-ACTA2 (Sigma, \#A5228, dilution 1:500), antiGAPDH (Sigma, \#G8795, dilution 1:1000), anti-HA (Santa Cruz, \#sc-805, dilution 1:1000), anti-MYC-tag (Abcam, \#9132, dilution 1:1000), anti-EP300 (Abcam, \#59240, dilution 1:1000), anti-actEP300 (ThermoFisher, PA5-64531, dilution 1:1000), anti-inaEP300 (Santa Cruz, \#130210, dilution 1:500), and anti-VIM (Cell Signaling, \#5741, dilution 1:500). Immunoreactive proteins were visualized with the corresponding horseradish peroxidase-conjugated secondary antibodies (Santa Cruz) using the Super Signal West Femto detection solutions (ThermoFisher Scientific). Signals were detected and analyzed with Luminescent Image Analyzer (Las 4000, Fujifilm). Protein concentrations were determined using Bradford substrate (Sigma).

miRNA fluorescence in situ hybridization. miRNA-FISH was performed as described earlier ${ }^{25}$ with minor adaptations. Briefly, primary cells were fixed with $4 \%$ paraformaldehyde (PFA), dehydrated with $70 \%$ ethanol, and incubated with pre-hybridization buffer ( $50 \%$ formamide, $5 \times$ SSC, $5 \times$ Denhardt's solution, $200 \mu \mathrm{g} /$ $\mathrm{ml}$ yeast RNA, $500 \mu \mathrm{g} / \mathrm{ml}$ salmon sperm DNA, and $2 \%$ Roche blocking reagent in DEPC-treated water). After $4 \mathrm{~h}$, pre-hybridization buffer was replaced with denaturizing hybridization buffer (10\% CHAPS, $20 \%$ Tween, $50 \%$ formamide, $5 \times$ SSC, $5 \times$ Denhardt's, $200 \mu \mathrm{g} / \mathrm{ml}$ yeast RNA, $500 \mu \mathrm{g} / \mathrm{ml}$ salmon sperm DNA, and $2 \%$ Roche blocking reagent in DEPC-treated water) containing biotin and/or digoxinlabeled Locked Nucleic Acid (LNA ${ }^{\mathrm{TM}}$ ) probes (Exiqon) specific to mature Mirlet7d, to a final concentration of $20 \mathrm{pM}$ and incubated at $55^{\circ} \mathrm{C}$ overnight. Next day, cells were briefly washed with $5 \%$ SSC buffer pre-warmed to $60^{\circ} \mathrm{C}$ and then incubated with $0.2 \%$ SSC at $60^{\circ} \mathrm{C}$ for $1 \mathrm{~h}$. Later, cells were incubated with B1 solution $(0.1 \mathrm{M}$ Tris $\mathrm{pH} 7.5,0.15 \mathrm{M} \mathrm{NaCl}$ ) at room temperature (RT) for $10 \mathrm{~min}$. B1 solution was then replaced with blocking solution (10\% FCS, $0.1 \mathrm{M}$ Tris pH 7.5, $0.15 \mathrm{M} \mathrm{NaCl})$ and incubated for $1 \mathrm{~h}$ at RT. Cells were then incubated with fluorescein isothiocyanate-labeled anti-Biotin (Abcam, \#53469) and/or Alexa fluor 647-labeled mouse anti-Digoxin (Dianova) antibodies overnight at $4{ }^{\circ} \mathrm{C}$. In order to couple mature Mirlet7d-FISH with EXOSC10 immunostaining, after completion of miRNA-FISH procedure, cells were incubated with mouse anti-EXOSC10 (Santa Cruz, \#374595, dilution 1:500) antibody overnight at $4{ }^{\circ} \mathrm{C}$ followed by Alexa Fluor 594-tagged anti-mouse secondary antibody (Invitrogen, Germany, dilution 1:2000) and 4',6-diamidino-2-phenylindole (DAPI) (Sigma, Germany) for $1 \mathrm{~h}$ at RT. DAPI was used as nuclear dye. Cells were examined with a confocal microscope (Zeiss). 
Immunohistochemistry and PLA assay. Immunostaining was performed as previously described ${ }^{17}$. Briefly, cells were grown on coverslips, fixed with $4 \%$ PFA for $10 \mathrm{~min}$ at RT, and permeabilized with $0.4 \%$ Triton X-100/1× PBS for $10 \mathrm{~min}$ at RT. During immunostaining procedure, all incubations and washes were performed with histobuffer containing $3 \%$ BSA and $0.2 \%$ Triton X-100 in $1 \times \mathrm{PBS}, \mathrm{pH}$ 7.4. Nonspecific binding was blocked by incubating with donkey serum and histobuffer $(1: 1(\mathrm{v} / \mathrm{v})$ ratio $)$ for $1 \mathrm{~h}$. Cells were then incubated with primary and then secondary antibodies for $1 \mathrm{~h}$ followed by nuclear staining. Immunostaining of cells were examined with a confocal microscope (Zeiss). Antibodies used were specific anti-ACTA2 (Sigma, \#A5228, dilution 1:500), anti-EP300 (Abcam, \#59240, dilution 1:500), anti-actEP300 (Biorbyt, \#34557, dilution 1:500), and anti-inaEP300 (Santa Cruz, \#130210, dilution 1:500). Alexa 488- or Alexa 594-tagged secondary antibodies (Invitrogen, Germany, dilution 1:2000) were used. DAPI (Sigma, Germany) was used as nuclear dye.

Paraffin-embedded lung tissue sections ( $3 \mu \mathrm{m}$ thick) were deparaffinized in xylene and rehydrated in a graded ethanol series to $\mathrm{PBS}(\mathrm{pH}$ 7.2). Antigen retrieval was performed by pressure cooking in citrate buffer ( $\mathrm{pH}$ 6.0) for $15 \mathrm{~min}$. Double immunofluorescence staining was performed with primary antibodies anti-ACTA2 (Sigma, \#A5228, dilution 1:500), anti-EP300 (Abcam, \#59240, dilution 1:500), antiactEP300 (Biorbyt, \#34557, dilution 1:500), and anti-inaEP300 (Santa Cruz, $\# 130210$, dilution 1:500). After overnight incubation with specific primary antibodies, slides were washed and incubated with the respective secondary antibodies, Alexa 488- and Alexa 555-conjugated goat anti-rabbit IgG (dilution 1:1000, Molecular Probes, Eugene, OR) for $1 \mathrm{~h}$. All sections were counterstained with nuclear DAPI (1:1000) and mounted with fluorescent mounting medium (Dako).

For PLA assay, cells were grown on coverslips, fixed, permeabilized, blocked, and incubated with primary antibodies as in immunostaining discussed above. After primary antibody incubation, the PLA was performed using the Duolink in situ Fluorescence Kit (Sigma, \#DUO92101-1KT) according to the manufacturer's instructions. The protein-protein interactions were analyzed by using confocal microscope (Zeiss). Primary antibody combination was goat antiHDAC1 (Santa Cruz, \#6298, dilution 1:500) and rabbit anti-AcK (Cell Signaling, \#9441 S, dilution 1:500). DAPI (Sigma, Germany) was used as nuclear dye.

HDAC activity assay. Assays were performed using the colorimetric HDAC activity assay from Bio Vision (Bio Vision Research Products, Mountain View, CA, USA) according to the manufacturer's instructions. Briefly, $50 \mu \mathrm{g}$ of nuclear extracts from Ctrl and IPF primary fibroblasts were diluted in $85 \mu \mathrm{l}$ of $\mathrm{ddH}_{2} \mathrm{O}$; then, $10 \mu \mathrm{l}$ of $10 \times$ HDAC assay buffer were added, followed by addition of $5 \mu \mathrm{l}$ of the colorimetric substrate; samples were incubated at $37^{\circ}$ for $1 \mathrm{~h}$. Subsequently, the reaction was stopped by adding $10 \mu \mathrm{l}$ of lysine developer and left for additional 30 $\min$ at $37^{\circ} \mathrm{C}$. Samples were then read in an enzyme-linked immunosorbent assay plate reader at $400 \mathrm{~nm}$. HDAC activity was expressed as relative OD values per $\mu \mathrm{g}$ of protein sample. The kit contains negative and positive controls that consist of nuclear extract of HeLa treated or not with Trichostatin A, respectively.

HDAC1-specific activity assay. Assays were performed using the HDAC1 fluorogenic assay kit from BPS Bioscience (Catalog \#50061) according to the manufacturer's instructions. In this assay, recombinant, full-length HDAC1 protein as a positive control and nuclear and cytoplasmic extracts from Ctrl and IPF primary fibroblasts were incubated with fluorophore-conjugated substrates, at a concentration equivalent to the substrate $\mathrm{Km}(6 \mu \mathrm{M}$ for HDAC1). Reactions were performed in assay buffer (50 mM HEPES, $100 \mathrm{mM} \mathrm{KCl}, 0.001 \%$ (v/v) Tween-20, $0.05 \%(\mathrm{w} / \mathrm{v}) \mathrm{BSA}, 200 \mu \mathrm{M}$ Tris (2-carboxyethyl)phosphine (TCEP) $\mathrm{pH} 7.4$ ) and followed for fluorogenic release of 7-amino-4-methylcoumarin from substrate upon deacetylase and trypsin enzymatic activity. Fluorescence measurements were obtained at $470 \mathrm{~nm}$ approximately every $5 \mathrm{~min}$ using a multilabel plate reader. Data were analyzed on a plate-by-plate basis for the linear range of fluorescence over time. The kit contains Trichostatin A as negative controls.

Migration and proliferation assays. Ctrl and IPF primary fibroblasts treated with EP300inh were grown till 90-95\% confluence in a six-well plate. Monolayers were scratched with a $200 \mu \mathrm{l}$ pipette tip (Biozym), washed to remove debris, and cultured to facilitate cell migration. Images were collected at the indicated time points using a $\times 5$ objective on a phase-contrast microscope (Leica DMI3000 B) to quantify cell migration distance at $12 \mathrm{~h}$ after scratch as computed by $\mathrm{D} 12 \mathrm{~h}=\mathrm{L} 12 \mathrm{~h}-\mathrm{L} 0 \mathrm{~h}$; where $\mathrm{D} 12 \mathrm{~h}$ and L12h are the net cell migration distance and the cell position at the $12 \mathrm{~h}$ time point, respectively. $\mathrm{LOh}$ is the original position ${ }^{55}$. Lung fibroblasts, to be assessed for cellular proliferation, were cultured either in 96- or 48-well plates. Fibroblast proliferation was determined using colorimetric bromodeoxyuridine incorporation assay kit (Roche, Germany) according to the manufacturer's instructions. Absorbance was measured at $370 \mathrm{~nm}$ with reference at $492 \mathrm{~nm}$ in a plate reader (TECAN, Germany). Depending on the experiment, proliferation of cells was plotted either as the difference of absorbance at 370 and $492 \mathrm{~nm}$ (A370 $\mathrm{nm}-\mathrm{A} 492 \mathrm{~nm}$ ) or as a percentage of absorbance compared with control cell absorbance.
Fibrosis score and collagen content. Fibrosis score was assessed using the Ashcroft score as previously described ${ }^{56}$; the grade (from 0 to 8 ) of fibrotic changes in histological lung samples were scaled numerically. Sections $(4 \mu \mathrm{m})$ of the left lobe of mice lungs were stained with hematoxylin and eosin. Whole sections were scanned with tenfold magnification using Leica DM6000B microscope (Leica, Germany). This resulted in around 25-40 pictures per mouse lung. Pictures from each mouse lung were graded, averaged, and depicted in a graph. Whole-lung lobe pictures were scanned with Nanozoomer 2.0 HT (Hamamatsu, Japan). Collagen content was assessed by Sirius red staining of lung sections and the area of collagen was measured using Leica image software (Leica Microsystems, Germany) according to the standard protocol.

Collagen assay. Total collagen content was determined using the Sircol Collagen Assay kit (Biocolor, Belfast, Northern Ireland). One lobe from the right lung was taken for analysis and the weight determined. Equal amounts of protein lysates were added to $1 \mathrm{ml}$ of Sircol dye reagent, followed by $30 \mathrm{~min}$ of mixing. After centrifugation at $10,000 \times g$ for $10 \mathrm{~min}$, the supernatant was carefully aspirated and $1 \mathrm{ml}$ of Alkali reagent was added. Samples and collagen standards were then read at $540 \mathrm{~nm}$ on a spectrophotometer (Bio-Rad). Collagen concentrations were calculated using a standard curve generated by using acid-soluble type 1 collagen.

Hydroxyproline measurements. Hydroxyproline levels in human lung fibroblasts and lung tissue were determined using the QuickZyme Hydroxyproline Assay kit (Quickzyme Biosciences, Leiden, the Netherlands). The cells and lung tissue were separately homogenized in $1 \mathrm{ml} 6 \mathrm{~N} \mathrm{HCl}$ with a Precellys tissue homogenizer $(2 \times$ $20 \mathrm{~s}, 3,800 \times g$ ). The homogenate was then hydrolyzed at $90^{\circ} \mathrm{C}$ for $24 \mathrm{~h}$. After centrifugation at $13,000 \times g$ for $10 \mathrm{~min}, 100 \mu \mathrm{l}$ from the supernatant was taken and diluted 1:2 with $4 \mathrm{~N} \mathrm{HCl}$. Thirty-five microliters of this working dilution was transferred to a 96-well plate. Likewise, a hydroxyproline standard $(12.5-300 \mu \mathrm{M})$ was prepared in $4 \mathrm{~N} \mathrm{HCl}$ and transferred to the microtiter plate. Following addition of $75 \mu \mathrm{l}$ of a chloramine T-containing assay buffer, samples were oxidized for 20 min at RT. The detection reagent containing $p$-dimethylaminobenzaldehyde was prepared according to the manufacturer's instruction and $75 \mu$ ladded to the wells After incubation at $60^{\circ} \mathrm{C}$ for $1 \mathrm{~h}$, the absorbance was read at $570 \mathrm{~nm}$ with a microtiter plate reader (Infinite M200 Pro, Tecan, Crailsheim, Germany) and the hydroxyproline concentration in the sample was calculated from the standard curve and related to the employed amount of lung tissue. The hydroxyproline content in lung tissue is given as $\mu \mathrm{g}$ hydroxyproline per mg lung tissue.

Experiments with mouse PCLS. PCLS were prepared from male, 12-16 weeks old C57BL/6 mice. Animals were humanely killed with mixture of ketamine and xylazine. A skin incision was performed on the ventral side of the neck and the trachea was exposed. A small incision was made on the trachea to allow insertion of an $18 \mathrm{G}$ catheter. Once the abdominal and chest cavities were opened, the collapsed lung lobes were infused with $1.5 \mathrm{ml}$ of warmed $\left(37^{\circ} \mathrm{C}\right) 1.5 \%$ agarose in Dulbecco's modified Eagle's medium (DMEM) (Gibco) through the inserted catheter. The agarose infusion was followed by $0.5 \mathrm{ml}$ of air to clear the airways of agarose. The whole mouse was placed at $4{ }^{\circ} \mathrm{C}$ for $10-15 \mathrm{~min}$ to allow the agarose to solidify. The lungs were then carefully dissected out of the chest cavity and kept in cold DMEM prior to slicing. After the selected lobes were separated, $8 \mathrm{~mm}$ lung tissue cores were prepared and sectioned into $250 \mu \mathrm{m}$-thick slices using a Krumdieck microtome (Alabama Research and Development, Munford, AL). PCLS were sorted serially during the slicing process, to ensure that every exposure group consisted of adjacent PCLS from throughout (distal, middle, and proximal regions) the core. After slicing, the sorted PCLS were incubated (3 PCLS per well in a 24-well plate) overnight in DMEM/Ham's F-12 Medium (Corning, Corning, New York) supplemented with $0.05 \mathrm{mg} / \mathrm{ml} \mathrm{Kanamycin} \mathrm{(Sigma-Aldrich,} \mathrm{St.} \mathrm{Louis,} \mathrm{MO),} \mathrm{1 \%} \mathrm{Gibco}$ Antibiotic-Antimycotic, containing 10,000 units $/ \mathrm{ml}$ of penicillin, $10 \mathrm{mg} / \mathrm{ml}$ of streptomycin, and $25 \mu \mathrm{g} / \mathrm{ml}$ of Amphotericin B (Life Technologies) at $37^{\circ} \mathrm{C}$.

Experiments with human PCLS. PCLS were prepared from tumor-free lung explants from patients who underwent lung resection for cancer at KRH Hospital Siloah-Oststadt-Heidehaus or the Hanover Medical School (both Hanover, Germany). Tissue was processed immediately within 1 day of resection as described before ${ }^{57}$. Briefly, human lung lobes were cannulated with a flexible catheter and the selected lung segments were inflated with warm $\left(37^{\circ} \mathrm{C}\right)$ low-melting agarose $(1.5 \%)$ dissolved in DMEM Nutrient Mixture F-12 Ham supplemented with L-glutamine 15 mM HEPES without phenol red, pH 7.2-7.4 (Sigma-Aldrich, Hamburg, Germany), $100 \mathrm{U} / \mathrm{mL}$ penicillin, and $100 \mu \mathrm{g} / \mathrm{mL}$ streptomycin (both from Biochrom, Berlin, Germany). After polymerization of the agarose solution on ice, tissue cores of a diameter of $8 \mathrm{~mm}$ were prepared using a sharp rotating metal tube. Subsequently, the cores were sliced into $300-350 \mu \mathrm{m}$ thin slices in DMEM using a Krumdieck tissue slicer (Alabama Research and Development, Munford, AL) PCLS were washed $3 \times$ for $30 \mathrm{~min}$ in DMEM and used for experiments. Viability of the tissue was assessed by a LDH Cytotoxicity Detection Kit (Roche, Mannheim, Germany) according to the manufacturer's instruction. Human PCLS were treated with Ctrl or pro-fibrotic cocktail as previously described ${ }^{58}$. 
RNA-seq and data analysis. RNA-seq of nuclear extracts from Ctrl and IPF primary fibroblasts was performed by Single End sequencing using Nextseq500 (Illumina) sequencer with V2 chemistry. RiboGone ${ }^{\text {rx }}$ - Mammalian (Clontech) was used to deplete ribosomal RNA from the samples. SMARTer ${ }^{\circledR}$ Universal Low Input RNA Kit for Sequencing (Clontech) was used for pre-amplification before library preparation. Library preparation was performed using the Low Input Libray Prep Kit (Clontech) following the manufacturer's instructions.

Raw reads were visualized by FastQC ${ }^{59}$ to determine the quality of the sequencing. Trimming was performed using trimmomatic ${ }^{60}$ with the following parameters LEADING:3 TRAILING:3 SLIDINGWINDOW:4:15 MINLEN:40 CROP:45 HEADCROP:5.

High quality reads were mapped to the NONCODE V4.0 (http://www.noncode. org/download.php) for the ncRNAs or hg19 genome using bowtie ${ }^{61}$ with -D 15 -R 3 -N 0 -L 20 -i S, 1, 0.56 -p8. After mapping, the sam files were converted to bam format by using samtools view (-Sb) followed by samtools sort. The sorted bam files were further converted to fastq files with SamToFastq.jar from picard-tools 1.119 (https://sourceforge.net/projects/picard/files/picard-tools/1.119/) and remapped to hg19 (UCSC reference genome). The tag libraries were created by using MakeTaglibrary from HOMER ${ }^{62}$ (-format sam). The makeUCSCfile command was used for visualizing the mapped sequence tag density after normalizing the samples to ten million. For the ncRNAs and for protein coding RNAs, samples were quantified by using analyzeRepeats.pl (http://homer.salk.edu/ homer/ngs/analyzeRNA.html) with the parameters (analyzeRepeats.pl database.gtf hg19 -count genes -strand both and -rpkm; reads per kilobase per millions mapped). The NONCODE_V.4.gtf file was used to quantify ncRNAs. KNIME 2.9.1 platform (Konstanz, Germany) was used to filter enriched ncRNAs and coding RNAs in the IPF compared with the Ctrl, by using the column filter, numeric binner, and row filter nodes.

UCSC genome browser was used to visualize Ctrl and IPF nuclear transcripts. Two scales were used for ncRNA and mRNA visualization.

Reporting summary. Further information on research design is available in the Nature Research Reporting Summary linked to this article.

\section{Data availability}

Sequencing data of nuclear RNA in Ctrl and IPF patients have been deposited in NCBI's Gene Expression Omnibus ${ }^{63}$ and is accessible through GEO Series with accession number GSE116086. RNA-seq data from total lung homogenates was retrieved through the GEO Series accession number GSE52463. The source data for all the plots and uncropped pictures of all the blots presented in the main and Supplementary Figures are provided as a Source Data file. Please direct any request for further information or reagents to Lead Contact Guillermo Barreto (guillermo.barreto@mpi-bn.mpg.de), LOEWE Research Group Lung Cancer Epigenetic, Max-Planck-Institute for Heart and Lung Research, Parkstrasse 1, 61231 Bad Nauheim, Germany.

Received: 5 September 2018 Accepted: 12 April 2019

Published online: 20 May 2019

\section{References}

1. Noble, P. W., Barkauskas, C. E. \& Jiang, D. Pulmonary fibrosis: patterns and perpetrators. J. Clin. Investig. 122, 2756-2762 (2012).

2. Coward, W. R., Saini, G. \& Jenkins, G. The pathogenesis of idiopathic pulmonary fibrosis. Ther. Adv. Respir. Dis. 4, 367-388 (2010).

3. Navaratnam, V. et al. The rising incidence of idiopathic pulmonary fibrosis in the U.K. Thorax 66, 462-467 (2011).

4. Swigris, J. J., Kuschner, W. G., Kelsey, J. L. \& Gould, M. K. Idiopathic pulmonary fibrosis: challenges and opportunities for the clinician and investigator. Chest 127, 275-283 (2005).

5. Nanthakumar, C. B. et al. Dissecting fibrosis: therapeutic insights from the small-molecule toolbox. Nat. Rev. Drug Discov. 14, 693-720 (2015).

6. Horowitz, J. C. \& Thannickal, V. J. Idiopathic pulmonary fibrosis: new concepts in pathogenesis and implications for drug therapy. Treat. Respir. Med. 5, 325-342 (2006).

7. Xia, H. et al. Identification of a cell-of-origin for fibroblasts comprising the fibrotic reticulum in idiopathic pulmonary fibrosis. Am. J. Pathol. 184, 1369-1383 (2014).

8. Andersson-Sjoland, A. et al. Fibrocytes are a potential source of lung fibroblasts in idiopathic pulmonary fibrosis. Int. J. Biochem. Cell Biol. 40 2129-2140 (2008).

9. El Agha, E. et al. Two-way conversion between lipogenic and myogenic fibroblastic phenotypes marks the progression and resolution of lung fibrosis. Cell Stem Cell 20, 261-273 e263 (2017).
10. Hung, C. et al. Role of lung pericytes and resident fibroblasts in the pathogenesis of pulmonary fibrosis. Am. J. Respir. Crit. Care Med. 188, 820-830 (2013)

11. Glasser, S. W. et al. Mechanisms of lung fibrosis resolution. Am. J. Pathol. 186 1066-1077 (2016).

12. Nicholson, A. G. et al. The relationship between individual histologic features and disease progression in idiopathic pulmonary fibrosis. Am. J. Respir. Crit. Care Med. 166, 173-177 (2002).

13. Allis, C. D. \& Jenuwein, T. The molecular hallmarks of epigenetic control. Nat Rev. Genet. 17, 487-500 (2016).

14. Berger, S. L. The complex language of chromatin regulation during transcription. Nature 447, 407-412 (2007).

15. Kouzarides, T. Chromatin modifications and their function. Cell 128, 693-705 (2007).

16. Stricker, S. H., Koferle, A. \& Beck, S. From profiles to function in epigenomics Nat. Rev. Genet. 18, 51-66 (2017).

17. Singh, I. et al. High mobility group protein-mediated transcription requires DNA damage marker gamma-H2AX. Cell Res. 25, 837-850 (2015).

18. Singh, I. et al. Hmga 2 is required for canonical WNT signaling during lung development. BMC Biol. 12, 21 (2014).

19. Ozturk, N., Singh, I., Mehta, A., Braun, T. \& Barreto, G. HMGA proteins as modulators of chromatin structure during transcriptional activation. Front Cell Dev. Biol. 2, 5 (2014).

20. Consortium, E. P. An integrated encyclopedia of DNA elements in the human genome. Nature 489, 57-74 (2012).

21. Rubio K., Dobersch S., Barreto G. Functional interactions between scaffold proteins, noncoding RNAs, and genome loci induce liquid-liquid phase separation as organizing principle for 3-dimensional nuclear architecture: implications in cancer. FASEB J. fj201802715R (2019).

22. Politz, J. C., Zhang, F. \& Pederson, T. MicroRNA-206 colocalizes with ribosome-rich regions in both the nucleolus and cytoplasm of rat myogenic cells. Proc. Natl Acad. Sci. USA 103, 18957-18962 (2006).

23. Politz, J. C., Hogan, E. M. \& Pederson, T. MicroRNAs with a nucleolar location. RNA 15, 1705-1715 (2009).

24. Rubio K., Dobersch S., Barreto G. Functional interactions between scaffold proteins, non-coding RNAs and genome loci induce liquid-liquid phase separation as organizing principle for three-dimensional nuclear architecture: implications in cancer. FASEB J. fj201802715R https://doi.org/10.1096/ fj.201802715R, (2019)

25. Singh I., et al. MiCEE is a ncRNA-protein complex that mediates epigenetic silencing and nucleolar organization. Nat. Genet. 50, 990-1001 (2018).

26. Thiagalingam, S. et al. Histone deacetylases: unique players in shaping the epigenetic histone code. Ann. N. Y. Acad. Sci. 983, 84-100 (2003).

27. Chen, J. \& Li, Q. Life and death of transcriptional co-activator p300. Epigenetics 6, 957-961 (2011).

28. Sanders, Y. Y. et al. Histone deacetylase inhibition promotes fibroblast apoptosis and ameliorates pulmonary fibrosis in mice. Eur. Respir. J. 43, 1448-1458 (2014)

29. Conforti, F. et al. The histone deacetylase inhibitor, romidepsin, as a potential treatment for pulmonary fibrosis. Oncotarget 8, 48737-48754 (2017)

30. Korfei, M. et al. Aberrant expression and activity of histone deacetylases in sporadic idiopathic pulmonary fibrosis. Thorax 70, 1022-1032 (2015).

31. Nance, T. et al. Transcriptome analysis reveals differential splicing events in IPF lung tissue. PLoS ONE 9, e92111 (2014)

32. Pandit, K. V. et al. Inhibition and role of let- $7 \mathrm{~d}$ in idiopathic pulmonary fibrosis. Am. J. Respir. Crit. Care Med. 182, 220-229 (2010).

33. Quinn, J. J. \& Chang, H. Y. Unique features of long non-coding RNA biogenesis and function. Nat. Rev. Genet. 17, 47-62 (2015).

34. Pefanis, E. et al. RNA exosome-regulated long non-coding RNA transcription controls super-enhancer activity. Cell 161, 774-789 (2015).

35. $\mathrm{Xu}, \mathrm{B}$. et al. Selective inhibition of EZH2 and EZH1 enzymatic activity by small molecule suppresses MLL-rearranged leukemia. Blood 125, 346-357 (2015).

36. Luo, Y. et al. Trans-regulation of histone deacetylase activities through acetylation. J. Biol. Chem. 284, 34901-34910 (2009).

37. Qiu, Y. et al. HDAC1 acetylation is linked to progressive modulation of steroid receptor-induced gene transcription. Mol. Cell 22, 669-679 (2006)

38. Adenuga, D., Yao, H., March, T. H., Seagrave, J. \& Rahman, I. Histone deacetylase 2 is phosphorylated, ubiquitinated, and degraded by cigarette smoke. Am. J. Respir. Cell Mol. Biol. 40, 464-473 (2009).

39. Kuzmichev, A., Nishioka, K., Erdjument-Bromage, H., Tempst, P. \& Reinberg, D. Histone methyltransferase activity associated with a human multiprotein complex containing the Enhancer of Zeste protein. Genes Dev. 16, 2893-2905 (2002).

40. Bose, D. A. et al. RNA binding to CBP stimulates histone acetylation and transcription. Cell 168, 135-149 e122 (2017). 
41. Huang, W. C. \& Chen, C. C. Akt phosphorylation of p300 at Ser-1834 is essential for its histone acetyltransferase and transcriptional activity. Mol. Cell Biol. 25, 6592-6602 (2005)

42. Bricambert, J. et al. Salt-inducible kinase 2 links transcriptional coactivator p300 phosphorylation to the prevention of ChREBP-dependent hepatic steatosis in mice. J. Clin. Investig. 120, 4316-4331 (2010).

43. Hammitzsch, A. et al. CBP30, a selective CBP/p300 bromodomain inhibitor, suppresses human Th17 responses. Proc. Natl Acad. Sci. USA 112, 10768-10773 (2015).

44. Saare, M., Rebane, A., Rajashekar, B., Vilo, J. \& Peterson, P. Autoimmune regulator is acetylated by transcription coactivator CBP/p300. Exp. Cell Res. 318, 1767-1778 (2012).

45. Rong, L. et al. Sirt 1 activator attenuates the bleomycin-induced lung fibrosis in mice via inhibiting epithelial-to-mesenchymal transition (EMT). Eur. Rev. Med. Pharm. Sci. 20, 2144-2150 (2016).

46. Zeng, Z. et al. Activation and overexpression of Sirtl attenuates lung fibrosis via P300. Biochem. Biophys. Res. Commun. 486, 1021-1026 (2017).

47. Rai, R. et al. A novel acetyltransferase p300 inhibitor ameliorates hypertensionassociated cardio-renal fibrosis. Epigenetics 12, 1004-1013 (2017).

48. Henderson, W. R. Jr. et al. Inhibition of Wnt/beta-catenin/CREB binding protein (CBP) signaling reverses pulmonary fibrosis. Proc. Natl Acad. Sci. USA 107, 14309-14314 (2010).

49. Dobersch S., Rubio K., Barreto G. Pioneer factors and architectural proteins mediating embryonic expression signatures in cancer. Trends Mol. Med. 25, 287-302 (2019).

50. Kodadek, T. Another one (of the "undruggable" targets) bites the dust: discovery of a potent and selective inhibitor of the histone acetyl transferase p300/CBP. Biochemistry 57, 899-900 (2018).

51. King, T. E. Jr. et al. A phase 3 trial of pirfenidone in patients with idiopathic pulmonary fibrosis. New Engl. J. Med. 370, 2083-2092 (2014).

52. Richeldi, L. et al. Long-term treatment of patients with idiopathic pulmonary fibrosis with nintedanib: results from the TOMORROW trial and its openlabel extension. Thorax 73, 581-583 (2018).

53. Kolb, M., Bonella, F. \& Wollin, L. Therapeutic targets in idiopathic pulmonary fibrosis. Respir. Med. 131, 49-57 (2017).

54. Percipalle, P. \& Obrdlik, A. Analysis of nascent RNA transcripts by chromatin RNA immunoprecipitation. Methods Mol. Biol. 567, 215-235 (2009).

55. Ilinskaya, O. N. et al. Direct inhibition of oncogenic KRAS by Bacillus pumilus ribonuclease (binase). Biochim. Biophys. Acta 1863, 1559-1567 (2016).

56. Al-Tamari, H. M. et al. FoxO3 an important player in fibrogenesis and therapeutic target for idiopathic pulmonary fibrosis. EMBO Mol. Med. 10, 276-293 (2018).

57. Wujak, L. et al. FXII promotes proteolytic processing of the LRP1 ectodomain. Biochim. Biophys. Acta 1861, 2088-2098 (2017).

58. Alsafadi, H. N. et al. An ex vivo model to induce early fibrosis-like changes in human precision-cut lung slices. Am. J. Physiol. Lung Cell Mol. Physiol. 312, L896-L902 (2017).

59. Patel, R. K. \& Jain, M. NGS QC Toolkit: a toolkit for quality control of next generation sequencing data. PLoS ONE 7, e30619 (2012).

60. Bolger, A. M., Lohse, M. \& Usadel, B. Trimmomatic: a flexible trimmer for Illumina sequence data. Bioinformatics 30, 2114-2120 (2014).

61. Langmead, B. \& Salzberg, S. L. Fast gapped-read alignment with Bowtie 2. Nat. Methods 9, 357-359 (2012)

62. Heinz, S. et al. Simple combinations of lineage-determining transcription factors prime cis-regulatory elements required for macrophage and B cell identities. Mol. Cell 38, 576-589 (2010).
63. Edgar, R., Domrachev, M. \& Lash, A. E. Gene Expression Omnibus: NCBI gene expression and hybridization array data repository. Nucleic Acids Res. 30, 207-210 (2002).

\section{Acknowledgements}

We thank Roswitha Bender, Uta Eule, and Natascha Wilker for technical support; Dr. Yi Qiu, College of Medicine, University of Florida, for acetyl-mimic HDAC1-Q and non-acetyl-mimic HDAC1-R constructs; Dr. Moisés Selman, Dr. Annie Pardo, and Dr. Johannes Graumann for helpful discussions. Guillermo Barreto was funded by the "LOEWE-Initiative der Landesförderung" (Wiesbaden, Germany) (III L 4-518/ 15.004 2009); the "Deutsche Forschungsgemeinschaft" (DFG, Bonn, Germany) (BA 4036/4-1), and the Excellence Cluster Cardio Pulmonary System (ECCPS, Giessen, Germany). K.R. received a doctoral fellowship from CONACyT-DAAD (PKZ91549687). T.B. was supported by the DFG (SFB 1213 TP A02 and KFO 309 TP 08 $\mathrm{Z} 1, \mathrm{Z} 2)$. This work was done according to the program of competitive growth of the Kazan Federal University and the Russian Government. G.B. is scientific advisor for Hawkeye Bio, Inc.

\section{Author contributions}

K.R., I.S., S.D., P.S., S.G., J.C., L.W., P.B. and G.B. designed and performed the experiments. G.B., I.S., K.R., R.S. and S.S.P. designed the study and analyzed the data. I.S., A.M. H.C.F., C.M.C., S.B., A.G., D.P.G., G.D., K.T.P., M.W., W.S., M.H. and T.B. were involved in study design. G.B., K.R., I.S., A.M., S.S.P., K.T.P., M.H. and T.B. wrote the manuscript. All authors discussed the results and commented on the manuscript.

\section{Additional information}

Supplementary Information accompanies this paper at https://doi.org/10.1038/s41467 019-10066-7.

Competing interests: The authors declare no competing interests.

Reprints and permission information is available online at http://npg.nature.com/ reprintsandpermissions/

Journal peer review information: Nature Communications thanks Dianhua Jiang and John Halsall for their contribution to the peer review of this work.

Publisher's note: Springer Nature remains neutral with regard to jurisdictional claims in published maps and institutional affiliations.

\begin{abstract}
Open Access This article is licensed under a Creative Commons $\mathrm{BY}$
Attribution 4.0 International License, which permits use, sharing,
adaptation, distribution and reproduction in any medium or format, as long as you give appropriate credit to the original author(s) and the source, provide a link to the Creative Commons license, and indicate if changes were made. The images or other third party material in this article are included in the article's Creative Commons license, unless indicated otherwise in a credit line to the material. If material is not included in the article's Creative Commons license and your intended use is not permitted by statutory regulation or exceeds the permitted use, you will need to obtain permission directly from the copyright holder. To view a copy of this license, visit http://creativecommons.org/ licenses/by/4.0/.
\end{abstract}

(c) The Author(s) 2019

\section{Karla Rubio (1) ${ }^{1}$, Indrabahadur Singh ${ }^{1,2}$, Stephanie Dobersch (10 ${ }^{1}$, Pouya Sarvari ${ }^{3}$, Stefan Günther ${ }^{4}$, Julio Cordero ${ }^{1,5,6}$, Aditi Mehta ${ }^{1,7}$, Lukasz Wujak ${ }^{8}$, Hector Cabrera-Fuentes (10) 8,9,10,11,12, Cho-Ming Chao ${ }^{13,14,15,16,17}$, Peter Braubach (1) 16,18,19, Saverio Bellusci ${ }^{10,13,14,15,16}$, Werner Seeger 3,15,17,20, Andreas Günther (1) 15,16,20,21, Klaus T. Preissner $8,10,15$, Malgorzata Wygrecka (10,15,16, Rajkumar Savai (10 3,15,16, Dulce Papy-Garcia ${ }^{22}$, Gergana Dobreva ${ }^{5,6}$, Mathias Heikenwalder ${ }^{2}$, Soni Savai-Pullamsetti ${ }^{3,15,16}$, Thomas Braun ${ }^{4,15}$ \& Guillermo Barreto (D) $1,10,15,16,22$}

${ }^{1}$ Lung Cancer Epigenetic, Max-Planck-Institute for Heart and Lung Research, Bad Nauheim 61231, Germany. ${ }^{2}$ Division Chronic Inflammation and Cancer (F180), German Cancer Research Center (DKFZ), Heidelberg 69120, Germany. ${ }^{3}$ Department of Lung Development and Remodeling, MaxPlanck-Institute for Heart and Lung Research, Bad Nauheim 61231, Germany. ${ }^{4}$ Department of Cardiac Development, Max-Planck-Institute for Heart 
and Lung Research, Bad Nauheim 61231, Germany. ${ }^{5}$ Anatomy and Developmental Biology, CBTM, Heidelberg University, Mannheim 68167, Germany. ${ }^{6}$ European Center for Angioscience (ECAS), Medical Faculty Mannheim, Heidelberg University, Mannheim 68167, Germany.

${ }^{7}$ Pharmaceutical Technology and Biopharmaceutics, Department of Pharmacy, Ludwig-Maximilians-University of Munich, Munich 81377, Germany. ${ }^{8}$ Faculty of Medicine, Biochemistry Institute, Justus Liebig University, Giessen 35392, Germany. ${ }^{9}$ National Heart Research Institute, National Heart Centre Singapore, Singapore 169609, Singapore. ${ }^{10}$ Institute of Fundamental Medicine and Biology, Kazan (Volga Region) Federal University, Kazan 420008, Russian Federation. ${ }^{11}$ Tecnologico de Monterrey, Centro de Biotecnologia-FEMSA, Monterrey 64849 NL, Mexico. ${ }^{12}$ Cardiovascular and Metabolic Disorders Program, Duke-National University of Singapore Medical School, Singapore 169609, Singapore. ${ }^{13}$ Chair for Lung Matrix Remodeling, Excellence Cluster Cardio Pulmonary System, Justus Liebig University, Giessen 35392, Germany. ${ }^{14}$ International Collaborative Center on Growth Factor Research, School of Pharmaceutical Sciences, Wenzhou Medical University and Institute of Life Sciences, Wenzhou University, Wenzhou, Zhejiang 325035, China. ${ }^{15}$ Member of the Excellence Cluster Cardio Pulmonary System (ECCPS), The Universities of Giessen and Marburg Lung Center (UGMLC), Giessen 35392, Germany. ${ }^{16}$ German Center of Lung Research (Deutsches Zentrum für Lungenforschung, DZL), UGMLC, Giessen 35392, Germany. ${ }^{17}$ Department of General Pediatrics and Neonatology, University Children's Hospital Giessen, Justus Liebig University, Giessen 35392, Germany. ${ }^{18}$ Institute for Pathology, Hanover Medical School, Hanover 30625, Germany. ${ }^{19}$ Biomedical Research in Endstage and Obstructive Lung Disease Hanover (BREATH) Research Network, Hanover 30625, Germany. ${ }^{20}$ Pulmonary and Critical Care Medicine, Department of Internal Medicine, Justus Liebig University, Giessen 35392, Germany. ${ }^{21}$ Agaplesion Lung Clinic Waldhof Elgershausen, Greifenstein 35753, Germany. ${ }^{22}$ Laboratoire Croissance, Réparation et Régénération Tissulaires (CRRET), CNRS ERL 9215, Université Paris Est Créteil, Université Paris Est, Créteil F-94000, France 